\title{
MESENCHYMAL STROMAL CELLS FOR ARTICULAR CARTILAGE REPAIR: PRECLINICAL STUDIES
}

\author{
P. Fernández-Pernas ${ }^{1,2,3}$, L. Barrachina ${ }^{4}$, M. Marquina ${ }^{1}$, C. Rodellar ${ }^{4}$, M.C. Arufe ${ }^{2,3, \S}$ and C. Costa ${ }^{1, *, \S}$ \\ ${ }^{1}$ Infectious Diseases and Transplantation Division, Institut d'Investigació Biomèdica de Bellvitge \\ (IDIBELL), L'Hospitalet de Llobregat, Barcelona, Spain \\ ${ }^{2}$ Cellular Therapy and Medicine Regenerative Group, Department of Physiotherapy, Medicine and \\ Biomedical Sciences, Instituto de Investigación Biomédica de A Coruña (INIBIC), A Coruña, Spain \\ ${ }^{3}$ Agrupación Estratégica CICA-INIBIC, Complexo Hospitalario Universitario de A Coruña (CHUAC), \\ Sergas, Universidade da Coruña, As Xubias, A Coruña, Spain \\ ${ }^{4}$ Laboratorio de Genética Bioquímica (LAGENBIO) - Instituto Agroalimentario de Aragón, IA2 - Instituto \\ de Investigación Sanitaria de Aragón, IIS - Universidad de Zaragoza, Zaragoza, Spain \\ ${ }^{\S}$ These authors contributed equally to this work
}

\begin{abstract}
Rheumatic diseases such as osteoarthritis $(\mathrm{OA})$ are a major social and economic burden because of the population aging and the lack of curative solutions. An effective cell therapy may be the best treatment option for OA and other cartilage diseases. However, the main cellular strategy used to repair articular cartilage, the transplantation of autologous chondrocytes, is limited to a small number of patients with traumatic lesions. The use of joint replacement after years of disease progression proves the great medical need in current practice. Mesenchymal stromal/stem cells (MSCs) provide an alternative cell source for cartilage regeneration due to numerous advantages, comprising relative ease to isolate and culture, chondrogenic capacity, and antiinflammatory effects. Initial clinical trials with MSCs have led to encouraging results, but many variables have to be considered to attain true amelioration of disease or repair (type and status of cartilage disease, source and conditions of cells, administration regime, combinatorial approaches). Particularly, allogeneic MSCs are an advantageous cellular product. The animal models chosen for preclinical evaluation are also relevant for successful translation into clinical practice. Considering the limitations in the field, rigorous comparative and validating studies in well-established animal models (including large animals) are still needed to set up the bases for additional clinical trials. The present review of studies performed in small and large animal models should help clarify the applicability of MSC-based therapies for articular cartilage repair.
\end{abstract}

Keywords: Mesenchymal stromal cells, mesenchymal stem cells, cartilage repair, osteoarthritis, animal models, immune modulation.

*Address for correspondence: Cristina Costa, IDIBELL, Hospital Duran i Reynals, Gran Via de L'Hospitalet 199, L'Hospitalet de Llobregat, 08908 Barcelona, Spain.

Telephone number: +34 932607355 Fax number: +34 932607426 Email: ccosta_valles@yahoo.com

Copyright policy: This article is distributed in accordance with Creative Commons Attribution Licence (http://creativecommons.org/licenses/by-sa/4.0/).

\section{List of Abbreviations}

\section{ACLT}

APC

AT-MSCs

bFGF

BM-MSCs

BMP

$\mathrm{CD}$

CIA

CIOA anterior cruciate ligament

transection

antigen-presenting cell

adipose-tissue-derived MSCs

basic fibroblast growth factor

bone-marrow-derived MSCs

bone morphogenetic protein

cluster of differentiation

collagen-induced arthritis

collagenase-induced OA
CM-CSF

CTLA4-Ig

ECM

FBS

GAGs

HGF

i.a.

i.p.

i.v.

IDO granulocyte-macrophage colonystimulating factor cytotoxic T-lymphocyte-associated protein 4 immunoglobulin extracellular matrix fetal bovine serum glycosaminoglycans hepatocyte growth factor intra-articular intraperitoneal intravenous indoleamine 2,3-dioxygenase 


$\begin{array}{ll}\text { IFN } & \begin{array}{l}\text { interferon } \\ \text { insulin-like growth factor-binding } \\ \text { protein }\end{array} \\ \text { IGFs } & \begin{array}{l}\text { insulin growth factors } \\ \text { interleukin }\end{array} \\ \text { IL } & \begin{array}{l}\text { inducible nitric oxide synthase } \\ \text { iNOS }\end{array} \\ \text { ISCT } & \text { International Society for Cellular } \\ \text { MCP-1 } & \text { monocyte chemoattractant protein } \\ \text { MHC } & \text { major histocompatibility complex } \\ \text { MIA } & \text { monoiodoacetate } \\ \text { MPCs } & \text { mesenchymal precursor cells } \\ \text { MSCs } & \text { mesenchymal stromal or stem cells } \\ \text { NO } & \text { nitric oxide } \\ \text { OA } & \text { osteoarthritis } \\ \text { PGE2 } & \text { prostaglandin E2 } \\ \text { PRP } & \text { platelet-rich plasma } \\ \text { RA } & \text { rheumatoid arthritis } \\ \text { TGF- } \beta & \text { transforming growth factor- } \beta \\ \text { TIMP-2 } & \text { TIMP metallopeptidase inhibitor } 2 \\ \text { TNF } & \text { tumor necrosis factor } \\ \text { VEGF } & \text { vascular endothelial growth factor } \\ & \end{array}$

\section{Introduction}

Human articular cartilage is affected by various rheumatic diseases, such as OA, and has a very limited capacity for regeneration. In Western countries, this type of diseases is a serious social and economic burden due to the aging population. Cell therapy is a promising treatment option for $\mathrm{OA}$, but the high levels of inflammatory cytokines and other catabolic factors present in pathological joints may inhibit the synthesis of new articular cartilage or destroy newly formed articular cartilage (Lopes et al., 2017; Sommaggio et al., 2016). In addition, traumatic injury of cartilage, if not appropriately treated, leads to the early development of OA (Kwon et al., 2019). Currently, the transplantation of autologous chondrocytes extracted from lowfunctional cartilage areas is the main cell therapy strategy used to regenerate cartilage, particularly for the filling of traumatic defects (Jones et al., 2019; Kwon et al., 2019). However, the extraction process is highly invasive and the expansion in culture of these cells leads to their dedifferentiation, negatively impacting upon the clinical outcome and limiting their applicability (Davies and Kuiper, 2019). In current practice, disease progresses in most cases, especially for OA patients. In this scenario, full joint replacement is conducted, providing evidence for the magnitude of the medical need. Furthermore, $\mathrm{RA}$ is also a pressing medical problem due to the limitations of current therapeutic options (Liu et al., 2019). Although with lower incidence than OA, it is a complex cartilage disease with a more systemic profile and affecting a younger population. RA is also the object of intense study and could benefit from cell therapies with high immunoregulatory activity. In summary, many hurdles still need to be addressed for the successful application of cell therapies for articular cartilage repair.

Rapid advances in the isolation of multipotent progenitor cells, routinely called MSCs, from various animal tissues and organs have been increasingly sought after for improving cellular therapies of cartilage repair (Afizah and Hui 2016). MSCs have shown good plasticity as they are able to differentiate towards multiple mesenchymal lineages, including chondrocytes, and have a proven anti-inflammatory effect (Ruiz et al., 2016). They lack the ethical complications of embryonic stem cells, are easy to isolate and expand in culture, and their collection is less damaging to the patient, allowing for the production of the necessary cells for therapy. Accordingly, MSCs are an attractive cell source for cartilage regeneration. Notably, MSCs are also being tested in combination with newly developed implantable scaffolds as a cell target/carrier for new therapeutic approaches (Kwon et al., 2019). Nevertheless, much more research is needed before feasible and widespread clinical application of MSCs becomes reality.

Encouraging results have been obtained from the initial clinical trials with MSCs (Ruiz et al., 2016). However, many variables have to be considered in MSC-based therapy before it can become a wellaccepted and efficacious clinical practice. This includes the type and status of cartilage disease, cell source (tissue of origin, autologous or allogeneic) and dose, cell passage and culture conditions, genetic modifications, administration route/s, as well as timing and frequency of cell infusion (Afizah and Hui 2016). In this regard, the use of allogeneic MSCs is of special interest as it allows for the generation of a pre-tested and off-the-shelf product with multiple advantages over autologous cells. Specifically, it allows for the banking of MSCs obtained from healthy donors enabling quick availability and avoiding the delay inherent to autologous cell expansion. Furthermore, its usage would overcome limitations associated with obtaining autologous MSCs from elderly patients or with genetic or metabolic disorders (Chen and Tuan, 2008; Marycz et al., 2016).

Choosing the right animal model/s for preclinical studies is also key for generating and extracting valuable information that allows establishing the therapeutic regimes (Lo Monaco et al., 2018). Small animal models have been used extensively for determining the behavior of MSCs under various conditions, but also pose many limitations. Rabbits, sheep and pigs are certainly of interest for studies of cartilage repair despite the higher cost; whereas horses could be considered to be both preclinical models, due to the similarity of equine and human cartilage (Ruiz et al., 2016), as well as potential beneficiaries of advanced cellular therapies owing to the high casualty of cartilage lesions in this species (Broeckx et al., 2019). Lastly, although restricted to a few studies in this area, the use of non-human primates is of very high preclinical value. The present 
study reviews the knowledge and major advances been made at the preclinical level in the use of MSCs for articular cartilage repair in the mentioned species, with an emphasis on allogeneic MSCs. The information considered most valuable from each of these species was selected to cover all the spectrum of available tools. Particularly, studies in small animal models in the last years have focused on the optimization of the therapeutic conditions and generated encouraging results. Nevertheless, in most cases, subsequent confirmation in larger models and validation is still pending. Thus, additional work is needed at this level to continue progressing, with the goal that the benefit observed finally translates into the clinical practice.

\section{Cartilage and disease}

\section{Articular cartilage}

The articular cartilage is a highly specialized tissue that lacks innervation and is avascular, obtaining nutrients by diffusion from the synovial fluid. It is formed mainly of water, different types of collagen (especially type II), proteoglycans and GAGs. Chondrocytes are the only cell type (showing a very low division frequency), represent $1-2 \%$ of the tissue volume and secrete all the components of the ECM. ECM renewal is extremely slow. The ECM consists mainly of a dense net made of collagen fibers combined with macroaggregates of hydrophilic proteins loaded with water molecules. Cyclic pressures act on cartilage, mobilizing water molecules within the matrix. ECM stabilization depends on glycoproteins and proteoglycans, as well as integrins present on chondrocytes. With aging, cartilage suffers several changes, including reduction in the number of chondrocytes and quality and quantity of proteoglycans. Senescent cartilage behaves worse against mechanical requirements, which is a potential origin of pathologies such as OA, being the very-close relationships between cartilage aging and arthritis disease (Poole et al., 2001).

Articular cartilage is organized into four different zones based on their functional and structural differences. The superficial zone lies next to the articular cavity and is in direct contact with the synovial fluid. Chondrocytes have a flattened morphology, produce thin horizontal collagen fibrils (Tallheden et al., 2006), and secrete lubricin to act as a lubricant in the joint (Flannery et al., 1999; Poole et al., 2001). Just below, the medial zone, with more oval and larger chondrocytes, contains more proteoglycans and has a lower cell density. The collagen fibers are thicker and more randomly distributed. Next, there is the deep or radial zone, with fibers oriented more perpendicularly to the surface and the chondrocytes disposed into a column-like structure in parallel with the collagen fibers. Located below, the tidemark is a thin layer or interface that separates the non-calcified cartilage from the calcified zone. The chondrocytes in this last layer secrete type $X$ collagen, a marker of hypertrophy responsible for the calcification of the ECM (Poole et al., 2001). Thus, this is a complex structure difficult to fully recover once it is lost.

\section{Lesions and therapies}

Cartilage integrity is essential for the proper function of the joint. The main problem is that, once injured, cartilage has a very low or even no repairing capacity. Most of the injuries are either due to mechanical trauma, also called focal lesion (very common in athletes), or to progressive degeneration, primary or secondary, as in the case of OA (Shi et al., 2017). OA is a multifactorial disease that leads to degradation of articular cartilage. Although the specific causes of the disease are still unknown, many risk factors have been identified, such as mechanical trauma, age, obesity, diabetes, inflammation, and genetics. After cartilage degradation, the subchondral bone gets exposed resulting in stiffness, inflammation, and pain (Alfredson et al., 1999; Lorentzon et al., 1998).

Inflammation itself is the cause of many of the symptoms. In pathological conditions, chondrocytes secrete a variety of inflammatory mediators such as proteases (collagenases, aggrecanases, etc.), pro-inflammatory cytokines (IL- $1 \alpha$, IL- $1 \beta$, TNF $\alpha$, IL-8, IL-17, IL-18), anti-inflammatory cytokines and antagonists (IL-4, IL-10, IL-13, IGFs, MCP-1, TGF- $\beta$, NO) etc. (Hunziker et al., 2002; O'Hara et al., 1990). Pieces of degraded cartilage are phagocyted by synovial cells, which also secrete pro-inflammatory mediators, leading to secretion of proteolytic enzymes and further cartilage degradation. This response is amplified by B cells, T cells and macrophages. Complement activation also plays a role in this process, contributing to cartilage inflammation, degradation, and OA (Sommaggio et al., 2013; Wang et al., 2011). On the other hand, the synovial membrane contributes to bone spur formation through BMP signaling (Sellam et al., 2010).

The goal of cartilage repair is to reconstitute the lesion with a tissue that has identical or at least very similar properties to the original cartilage, including integration into surrounding tissue. To date, there is no treatment that fulfils these requirements, although some therapies improve the patients' experience. Non-surgical treatments of the symptoms include standard analgesic and anti-inflammatory treatments (Vista et al., 2011), as well as dietary supplements such as chondroitin sulfate. The most common surgical treatment is the total joint replacement by means of a prosthesis once the function is lost or pain is unbearable. However, other methods, such as debridement (Moseley et al., 2002) or osteochondral auto and allograft (McCoy and Miniaci et al., 2012), have been used. Regarding cell-based treatments, autologous chondrocyte implantation (used either alone or in combination with scaffolds) is the approach clinically approved for the filling of chondral defects and is considered to produce the best outcomes (Davies and Kuiper 2019; Riboh 
et al., 2017; Shanmugaraj et al., 2019). Furthermore, chondrocytes are also of interest for the development of cell therapies for OA (Cherian et al., 2015; Sato et al., 2019; Schinhan et al., 2013). The use of multipotent or stem cells is a promising way to eliminate the need for a cartilage biopsy and an attractive choice for targeting cartilage diseases with an inflammatory component such as OA and RA. In the case of MSCs, the results are promising, but superiority to other treatments in animal studies is not consistent (Dahlin et al., 2014; Kwon et al., 2019, Liu et al., 2019; Xing et al., 2018). Thus, more stringently designed studies are needed, first at the preclinical and then at the clinical level to show significant improvement or at least non-inferiority. A scheme of OA, therapeutic targets, current treatments, and therapies in development is shown in Fig. 1.

\section{MSCs}

According to the standard definition of MSCs by the ISCT (Dominici et al., 2006), these clonal cells adhere to plastic, express CD markers such as CD73, CD90, and CD105, and can differentiate into adipogenic, chondrogenic, and osteogenic lineages in vitro. Particularly, these multipotent fibroblast-like cells can be found in almost all tissues and can differentiate towards bone (Tawonsawatruk et al., 2012), cartilage (Yeh et al., 2013), muscle (Park et al., 2016), tendon, ligament (Liang et al., 2013), fat (Contador et al., 2015), and a variety of other connective tissues (Kil et al., 2016; Ullah et al., 2016) (Fig. 2). MSCs display high self-renewal capacity, a process by which a stem cell divides asymmetrically or symmetrically forming one or two daughter stem cells with a similar potential to the mother cell (Wang et al., 2013), while, simultaneously, maintaining pluripotency (Jiang et al., 2002). However, isolated MSCs have been reported to vary in their potency and self-renewal potential. As a result, the MSCs used for clinical applications often lead to variable or even conflicting results.

MSCs have been isolated from many different adult tissues, including bone marrow (Karaoz et al., 2009), adipose tissue (Blazquez-Martinez et al., 2014), synovial membrane (De Bari et al., 2001), connective tissues of dermis (Manini et al., 2011), skeletal muscle (Almeida and O'Brien, 2013), peripheral blood (Trivanović et al., 2013), liver (D'souza et al., 2015), lung (Gong et al., 2014), blood vessels (Pacini and Petrini, 2014) as well as from rather "young sources" such as amniotic fluid (Pappa and Anagnou, 2009), amniotic membrane (Díaz-Prado et al., 2011), umbilical cord blood (Secco et al., 2008), umbilical cord stroma (Fernández-Pernas et al., 2016), or placenta (Pelekanos et al., 2016). In recent years, the number of tissues with a potential for tissue engineering has increased (Rossignoli et al., 2013). Notably, MSCs can differentiate both in vivo and in vitro into various mesenchymal cells and exhibit remarkable plasticity, given their ability to trans-differentiate towards different lineages (Jeon et al., 2016; Jin et al., 2013). However, only a small percentage of the injected MSCs differentiate and migrate in vivo, which would not justify the therapeutic effects observed (Murphy et al., 2003). The focus on MSC therapeutic ability is currently being moved to their regulatory properties, since MSCs can interact with their environment and elicit trophic, proangiogenic, and anti-inflammatory effects. By cross-talking with immune cells by both direct cell-cell contact and paracrine signaling, MSCs promote regulation of the catabolic environment of the diseased joint. This paradigm shift is highly relevant, meaning that MSCs would not only be 'building blocks' but actually have the ability to direct the healing process (Barry and Murphy, 2013).

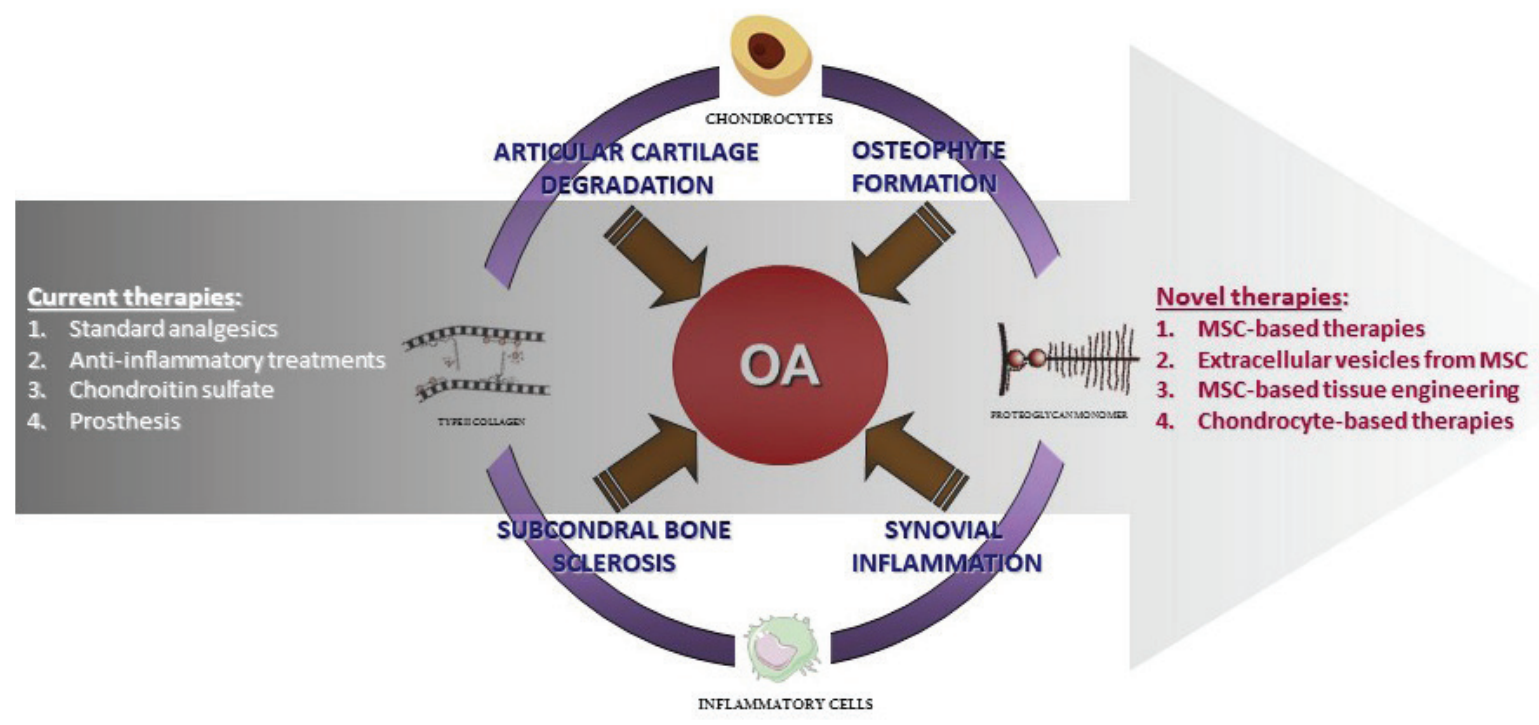

Fig. 1. Scheme of OA with potential therapeutic targets, current and future therapies. Both physiological and immunological alterations should be addressed for the development of a successful and efficacious OA therapy. Renewal of the cellular and ECM compartments is needed for the formation of high-quality hyaline cartilage at the proper anatomical site and full functional recovery. 


\section{Paracrine effects and immunomodulation}

MSCs have the capacity to secrete a wide variety of cytokines, chemokines, and growth factors. Several studies based on examination and modulation of the MSC secretome in vivo have identified high levels of proteins involved in the immune response such as IL-6, IL-8, MCP-1, and TGF- $\beta$; ECM remodelers such as TIMP-2, fibronectin, periostin, collagen, decorin, and metalloproteinase inhibitors; growth factors and their regulators such as VEGF, CM-CSF, BMP-2, bFGF, as well as IGFBP3, 4, and 7 (Elahi et al., 2016) (Fig. 2). Notably, the MSC secretome, either directly or through extracellular vesicles, influences cartilage regeneration, especially by the release of TGF- $\beta$-superfamily proteins (Lo Monaco et al., 2018). This process contributes to cartilage repair mostly by stimulating endogenous cells and promoting the deposition of collagen type II and glycosaminoglycans (Murphy et al., 2003; Zhang et al., 2016).

MSCs can modulate the immune system and are effective for the treatment of various immune response disorders in both human and animal models (de Miguel-Beriain et al., 2015; Jung et al., 2012; Li et al., 2009). The underlying mechanism of immune modulation is not fully understood. However, there is evidence for both cell-to-cell contact mechanisms and release of soluble immunosuppressive factors. They interact with a broad range of immune cells and avert the excessive response of T and B cells, dendritic cells, macrophages, and natural killer cells (Chao et al., 2008; Jung et al., 2012). Furthermore, MSCs can also induce regulatory $\mathrm{T}$ cells and maintain their suppressive activity on self-reactive T-effector responses (Chen et al., 2004). In recent years, it was proposed that MSCs interact with their environments both by negatively regulating the immune response, in case of major inflammation, or stimulating the immune response system by releasing pro-inflammatory molecules when the level of inflammatory cytokines is low (Marquina et al., 2017).

Regarding their migratory capacity, MSCs have been reported to reach damaged tissue in response to a combination of signaling molecules coming from the injured tissue. Homing-related molecules in general can be up-regulated by inflammatory

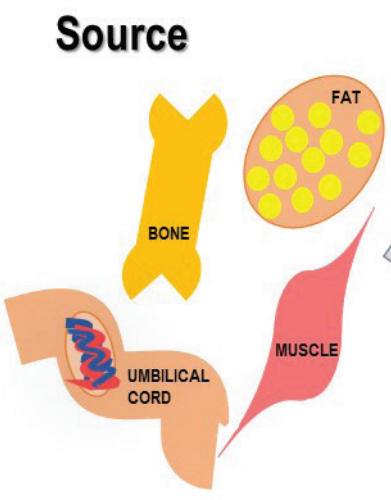

\section{Surface markers}
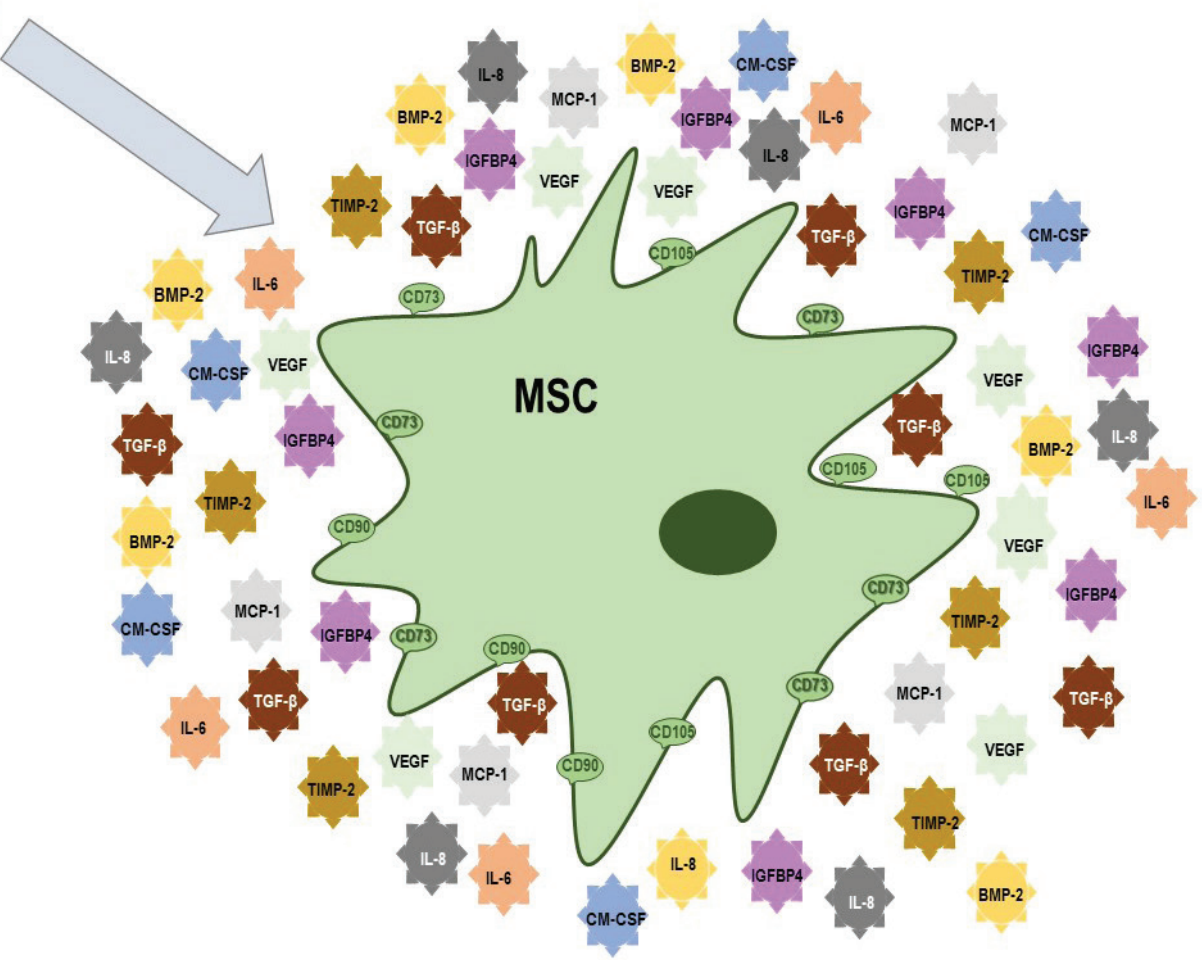
cytokines such as TNFa and IL-1 (Tanaka, 2015), suggesting that different inflammation states might promote distinct MSC engraftment and therapeutic efficiencies (Chen and Tuan, 2008). Particularly, expression of hyaluronic acid and its receptor CD44 by MSCs may be involved in the migration of MSCs to cartilage defects (Lo Monaco et al., 2018).

\section{Preclinical studies of articular cartilage repair by MSCs}

In agreement with the progress of advanced therapies for cartilage repair, an effort has been made during the last decade towards the preclinical assessment of MSC-based therapies. This research has involved a wide variety of animal models, comprising both small and large animals, multiple disease indications and procedures (i.e. traumatic injury of various joints, induced or spontaneous OA, and RA), as well as different MSC origins and administration regimes (Table 1-3). The large amount of information available, sometimes contradictory, justifies a thorough appraisal of this topic. Notably, an extensive review of the factors under consideration for choosing one or another species has been recently conducted (Lo Monaco et al., 2018).

Interest in MSCs for treating joint injuries was firstly raised because of the differentiation ability of these cells, assuming that MSCs would differentiate into chondrocytes to replace the damaged articular cartilage. Nevertheless, their regulatory features are now receiving more attention as these might be key for providing clinical benefit. The ability of MSCs to inhibit proliferation and regulate function of different immune cells in vitro has been demonstrated in different species (Carrade et al., 2013). Notably, variations in the immunomodulatory capacity of MSCs from the different species as compared to human MSCs should also be considered (Carrade and Borjesson 2013; Su et al., 2014). In general, large animals, being more similar to humans, provide higher value for preclinical studies, but their use is more costly and restricted.

Mediators secreted by MSCs also vary between tissue sources (Carrade et al., 2013), influencing their immunoregulatory activities and therapeutic efficacy. Recent experience in horses could provide relevant information in this respect. Equine MSCs from a hematic source produce NO but MSCs from solid tissues do not (Carrade et al., 2013). It is not clear to what extent the differences among secretory profiles in vitro may influence the MSC therapeutic efficacy in vivo. Nevertheless, it might explain the improved healing observed in tendinopathies treated with UC-MSCs and BM-MSCs that displayed superiority over AT-MSCs (Carrade et al., 2013; Romero et al., 2017). Some molecules, such as IDO and iNOS, are not expressed or secreted in basal conditions but are activated upon inflammatory stimulation (Barrachina et al., 2017), whereas other mediators such as TGF- $\beta 1$ and HGF are constitutively produced (Barrachina et al., 2016; Carrade et al., 2012; De Schauwer et al., 2014). Since inflammatory priming may be needed to induce MSC full regulatory function, stimulating MSCs with proinflammatory cytokines prior to in vivo administration is an interesting strategy to improve their therapeutic potential (Cuerquis et al., 2014).

Both autologous and allogeneic cells display similar immunomodulatory properties (Colbath et al., 2017b; Ranera et al., 2016) and, thus, would be equally able to modulate inflammation in joint pathologies. However, concerns are rising about the immunogenicity of allogeneic MSCs. Thus, an additional highly relevant paradigm change is the concept that MSCs are not truly immune-privileged but immune-evasive (Ankrum et al., 2014) (Fig. 3). In fact, the expression level of MHC-I and II molecules in MSCs is not static but regulated by conditions such as inflammation (Barrachina et al., 2017; Chan et al., 2008) and differentiation (Barrachina et al., 2018a; Lohan et al., 2014) (Fig. 3b). In addition, MHC matching between donor and receptor probably determines the production of cellular and humoral immune responses (Barrachina et al., 2020; Beggs et al., 2006; Berglund et al., 2017; Pezzanite et al., 2015; Poncelet et al., 2007), potentially limiting the repeated administration of allogeneic MSCs (Fig. 3a).

Repetitive MSC administration has been suggested to improve their therapeutic potential in joint pathologies (Hatsushika et al., 2014) since their lifespan in vivo appears to be short, especially for the allogeneic ones (Ryan et al., 2014). However, allogeneic studies are mainly focused on single administration, especially in large-animal models. In these models, repeated administration has been studied in healthy joints as proof of concept for their safety, but few studies have used repeated i.a. administration of allogeneic MSCs in pathological joints (Barrachina et al., 2018b; Magri et al., 2019). So far, single or repeated i.a. administration of allogeneic MSCs has been shown to be safe, although a slight-to-mild transient inflammatory reaction is occasionally observed (Ardanaz et al., 2016; Broeckx et al., 2014b). However, this type of response has also been found when using autologous cells and it has been hypothesized to be due to the high sensitivity of the joint or because potential FBS xeno-contamination (Ardanaz et al., 2016; Carrade et al., 2011; Pigott et al., 2013a; Pigott et al., 2013b). Therefore, a potential xenogeneic response raised by bovine proteins used for culture supplementation is another variable to account for in preclinical models on the way to develop safe treatments (Joswig et al., 2017). Alternatives, such as platelet lysate, are thus being investigated (Iudicone et al., 2014).

Future studies are needed to clarify the implications of both immunomodulation and immunogenicity in therapy with MSCs to treat joint pathologies. How these factors affect their chondrogenic capacity is also highly relevant, but this question remains mainly unanswered. 


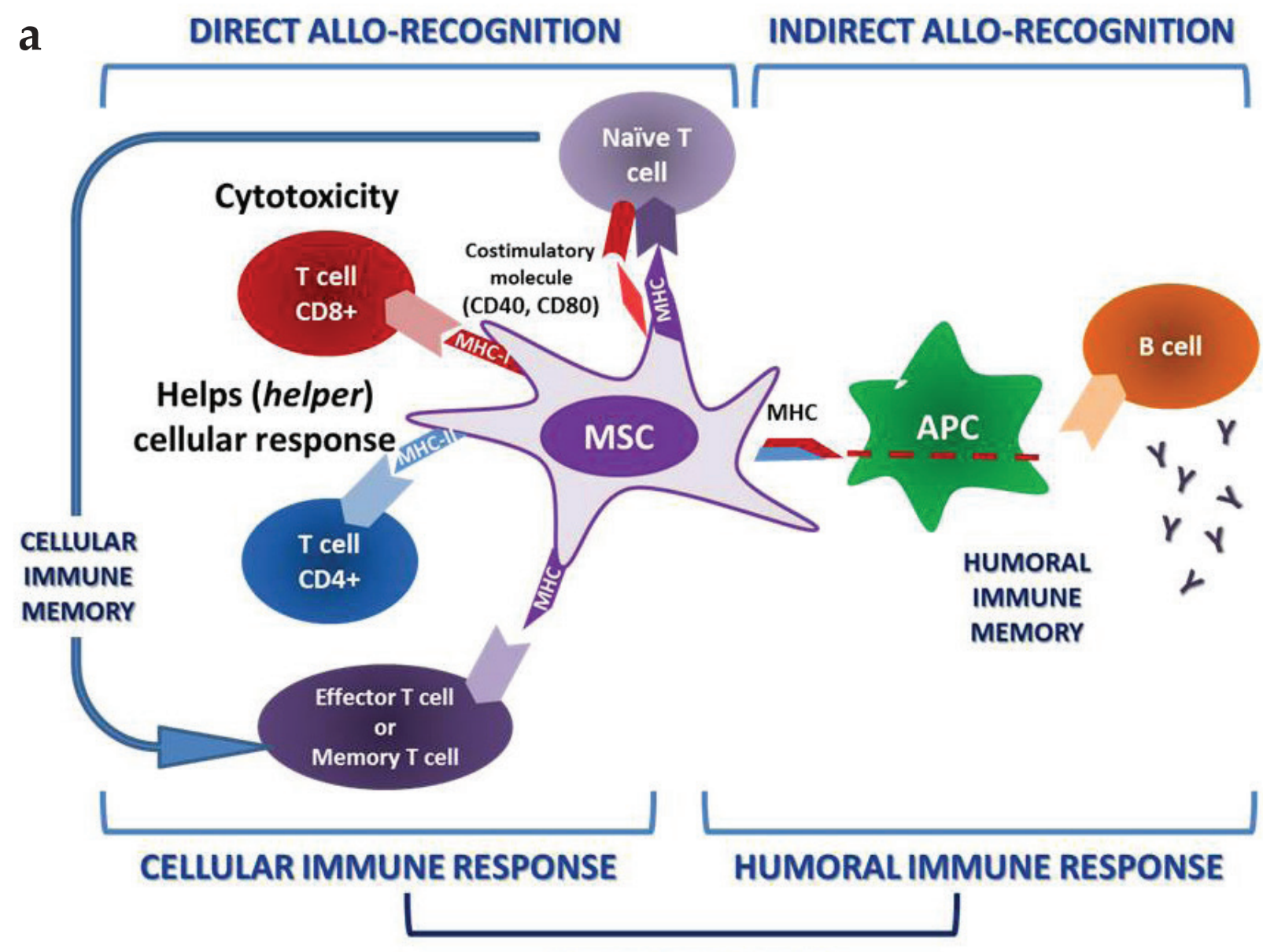

IMMUNE MEMORY

b

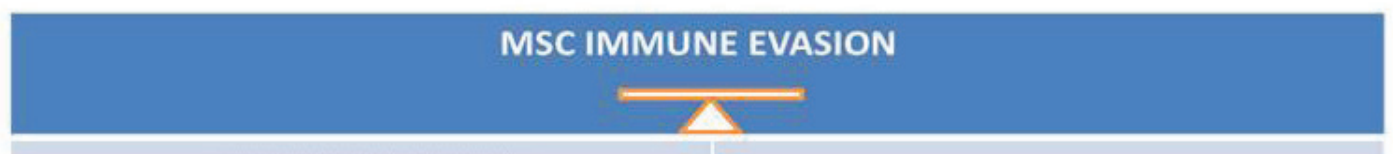

IMMUNOGENICITY

\begin{tabular}{l|c|c|c|}
$\begin{array}{l}\text { Expression } \\
\text { level MHC }\end{array}$ & $\begin{array}{c}\text { Expression level } \\
\text { costimulatory molecules }\end{array}$ & IMMUNOMODULATION
\end{tabular}

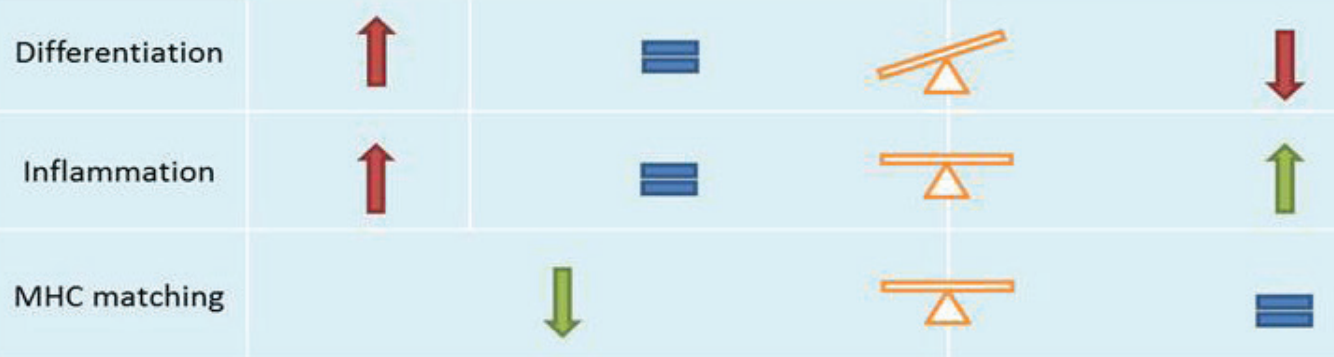

Fig. 3. Immune recognition of MSCs. (a) Schematic representation of how MSCs can be directly or indirectly recognized and develop both cellular and humoral immune responses, thus potentially leading to immune memory that would limit repeated administration. (b) Summary of current knowledge regarding the different factors affecting the balance between immunogenicity and immunomodulation of MSCs and thus, their immune evasive ability. Up and down arrows represent increase and decrease, respectively; equal symbol: no relevant change. 


\begin{tabular}{|c|c|c|c|c|c|c|c|c|c|c|c|c|c|c|c|c|c|c|c|}
\hline 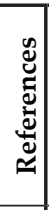 & 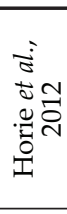 & 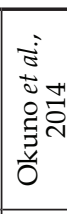 & 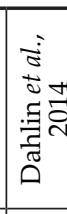 & 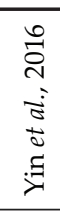 & 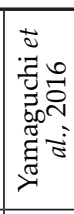 & 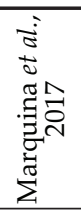 & 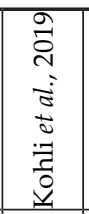 & 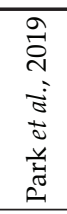 & 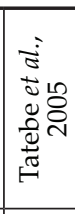 & 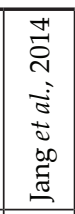 & 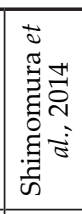 & 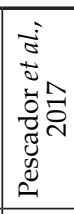 & 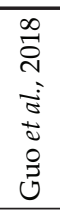 & 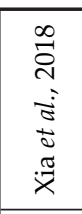 & 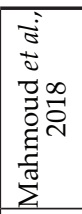 & 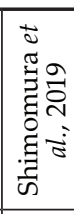 & 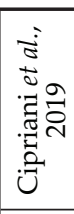 & 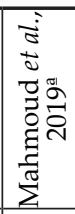 & 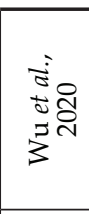 \\
\hline $\begin{array}{l}\text { : } \\
\text { : } \\
\vdots \\
0\end{array}$ & 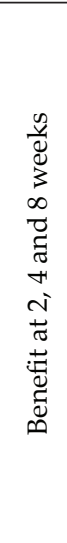 & 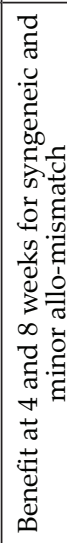 & 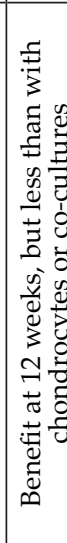 & 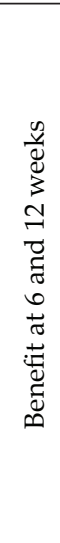 & 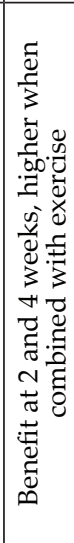 & 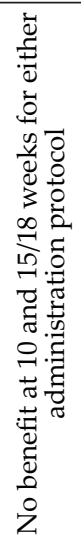 & 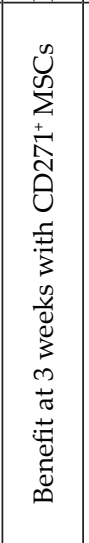 & 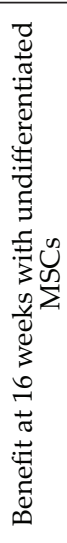 & 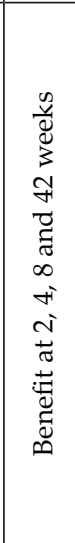 & 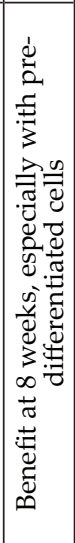 & 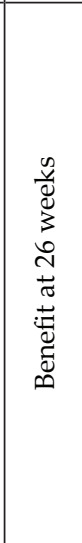 & 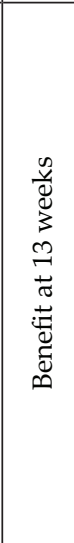 & 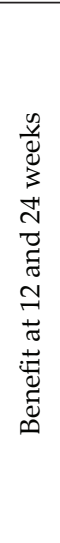 & 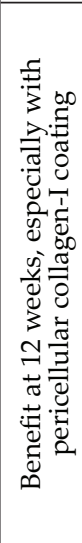 & 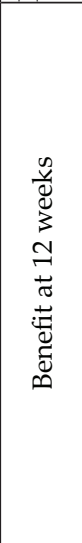 & 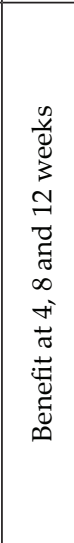 & 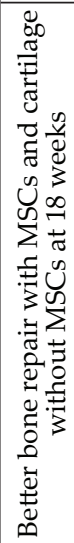 & 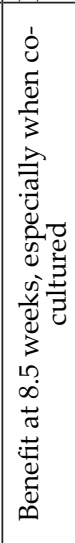 & 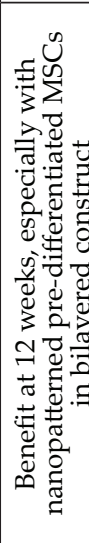 \\
\hline 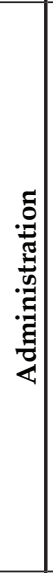 & 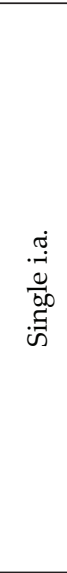 & 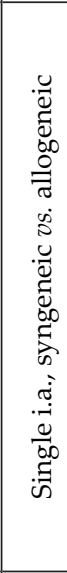 & 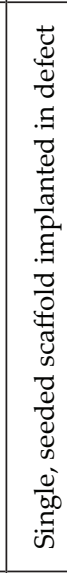 & 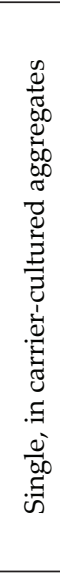 & 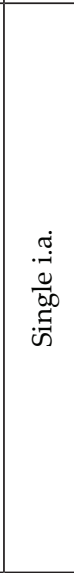 & 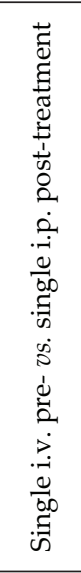 & 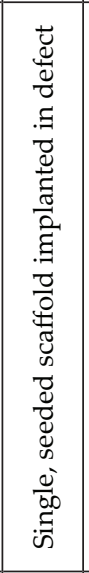 & 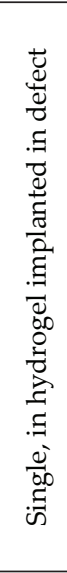 & 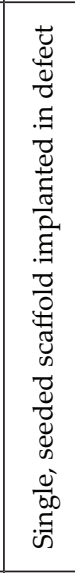 & 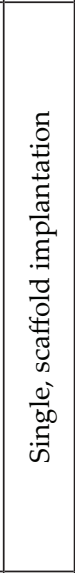 & 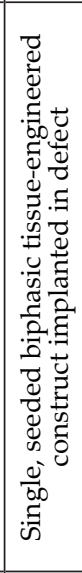 & 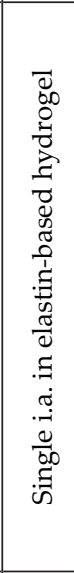 & 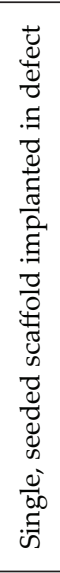 & 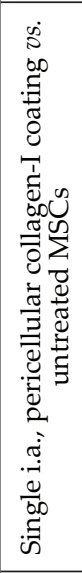 & 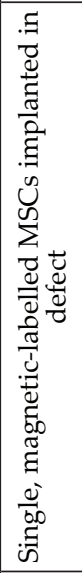 & 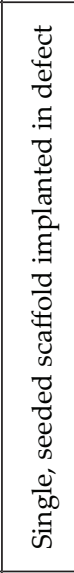 & 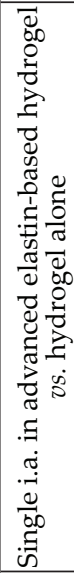 & 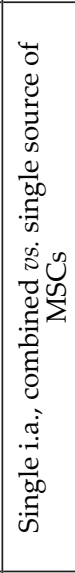 & 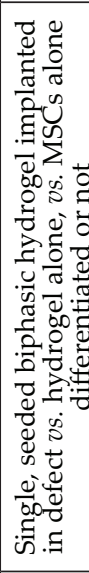 \\
\hline 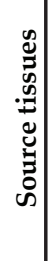 & 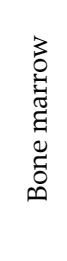 & 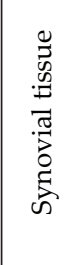 & 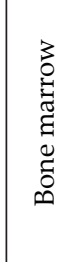 & 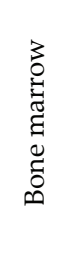 & 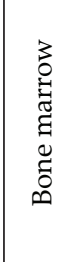 & 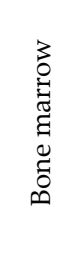 & 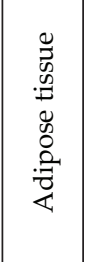 & 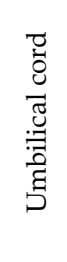 & 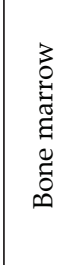 & 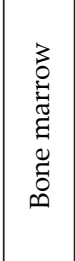 & 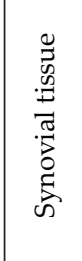 & 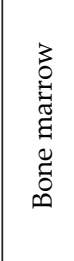 & 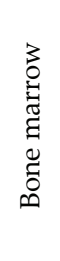 & 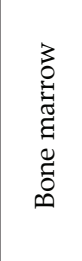 & 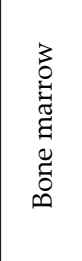 & 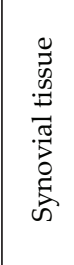 & 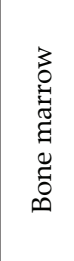 & 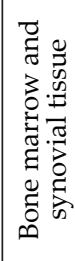 & 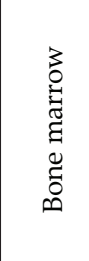 \\
\hline 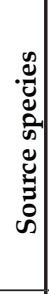 & 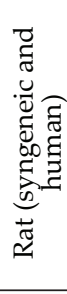 & 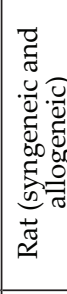 & 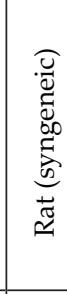 & 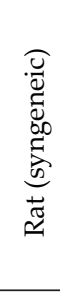 & 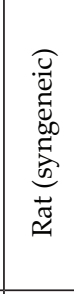 & 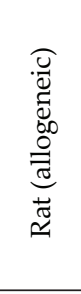 & 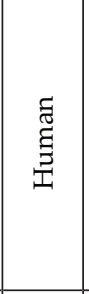 & 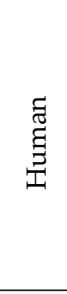 & 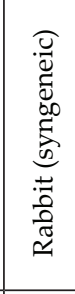 & 㞼 & 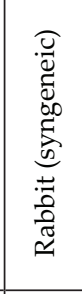 & $\begin{array}{l}\text { ฮ } \\
\text { ఏ్ } \\
\text { 至 }\end{array}$ & 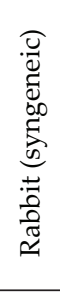 & 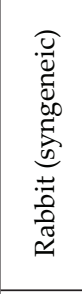 & 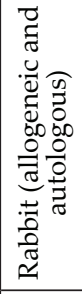 & 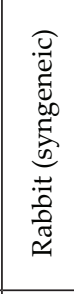 & 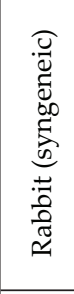 & 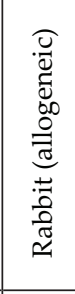 & 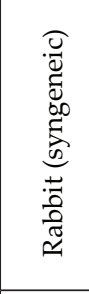 \\
\hline $\begin{array}{l}\bar{\Xi} \\
\bar{c} \\
\bar{\Sigma}\end{array}$ & 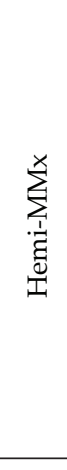 & 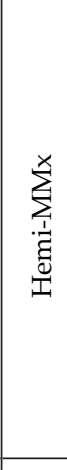 & 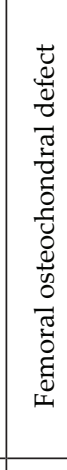 & 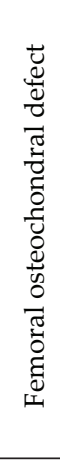 & 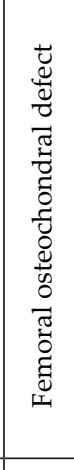 & 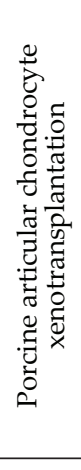 & 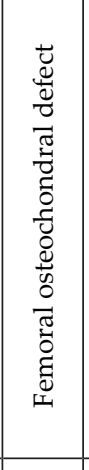 & 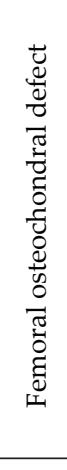 & 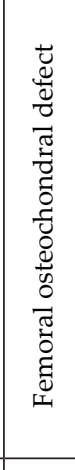 & 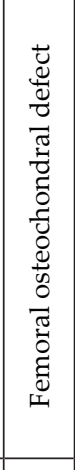 & 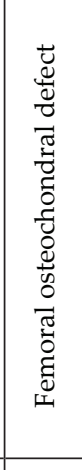 & 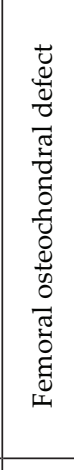 & 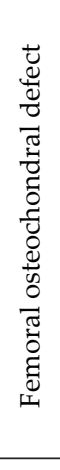 & 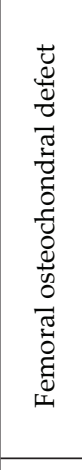 & 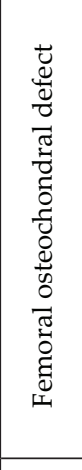 & 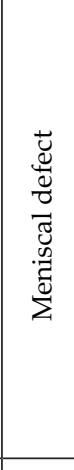 & 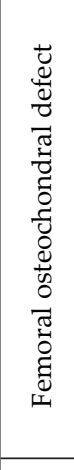 & 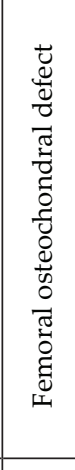 & 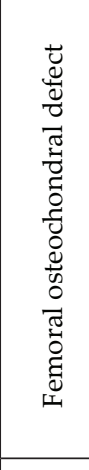 \\
\hline 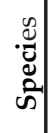 & $\overrightarrow{\widetilde{\Xi}}$ & 苑 & 芯 & 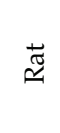 & 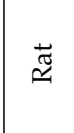 & $\overrightarrow{\widetilde{\Xi}}$ & 忒节 & 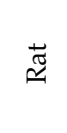 & $\begin{array}{l}\overrightarrow{0} \\
\overrightarrow{0} \\
\overrightarrow{0} \\
\approx\end{array}$ & 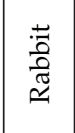 & 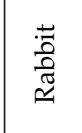 & 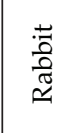 & 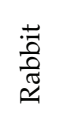 & 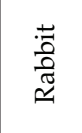 & 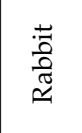 & 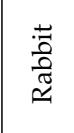 & 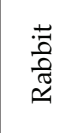 & 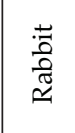 & 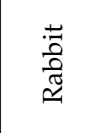 \\
\hline
\end{tabular}




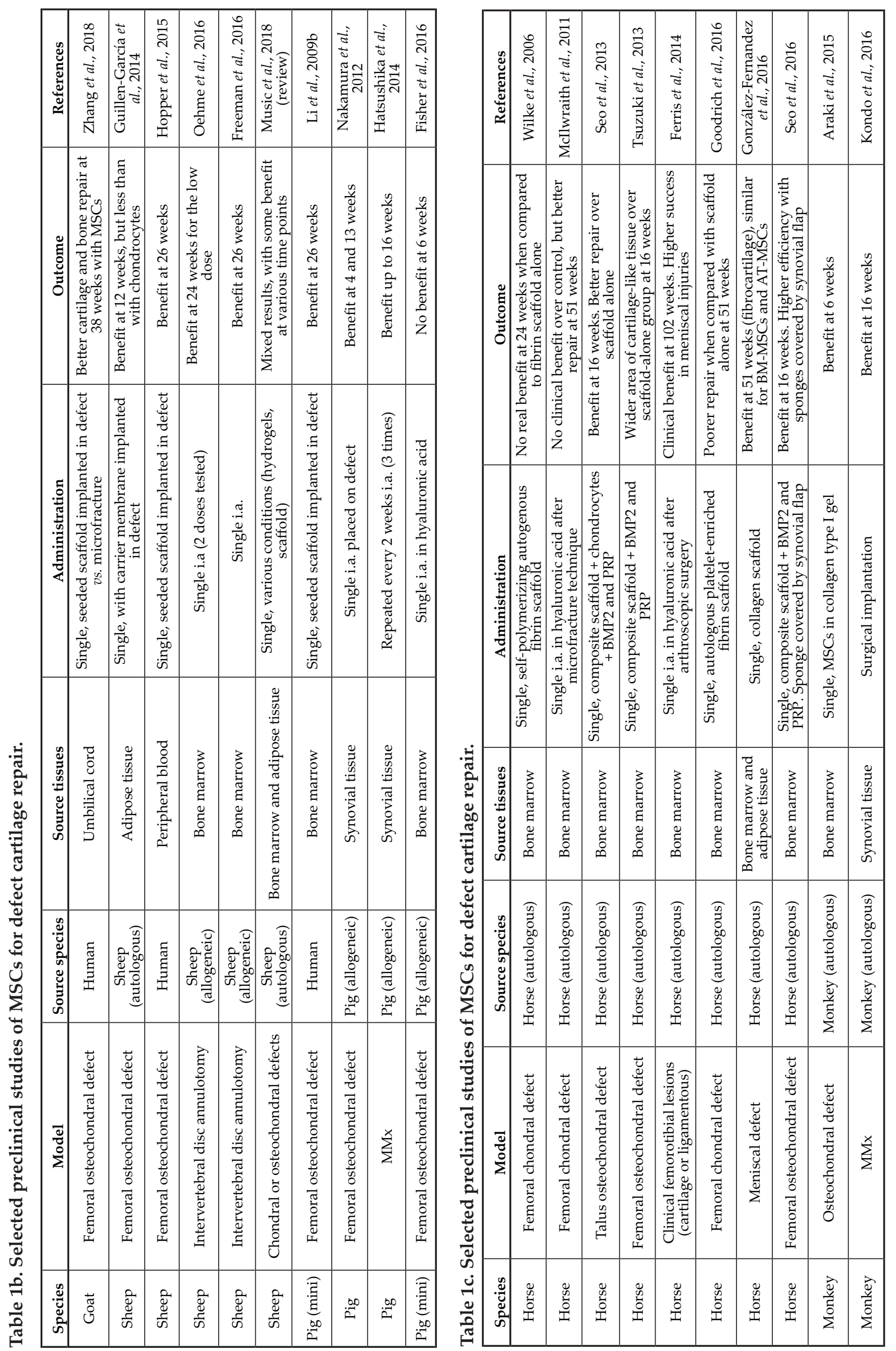




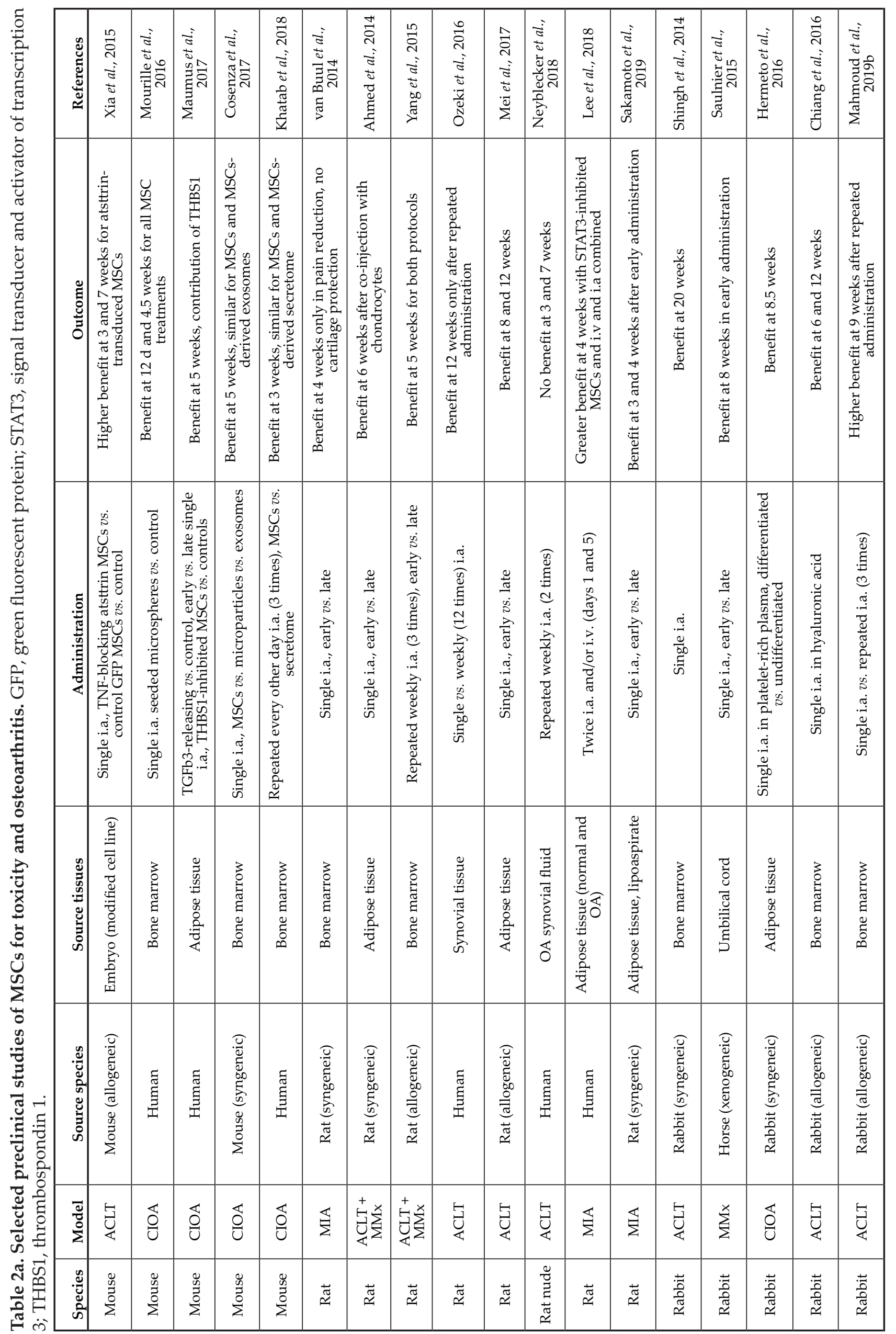




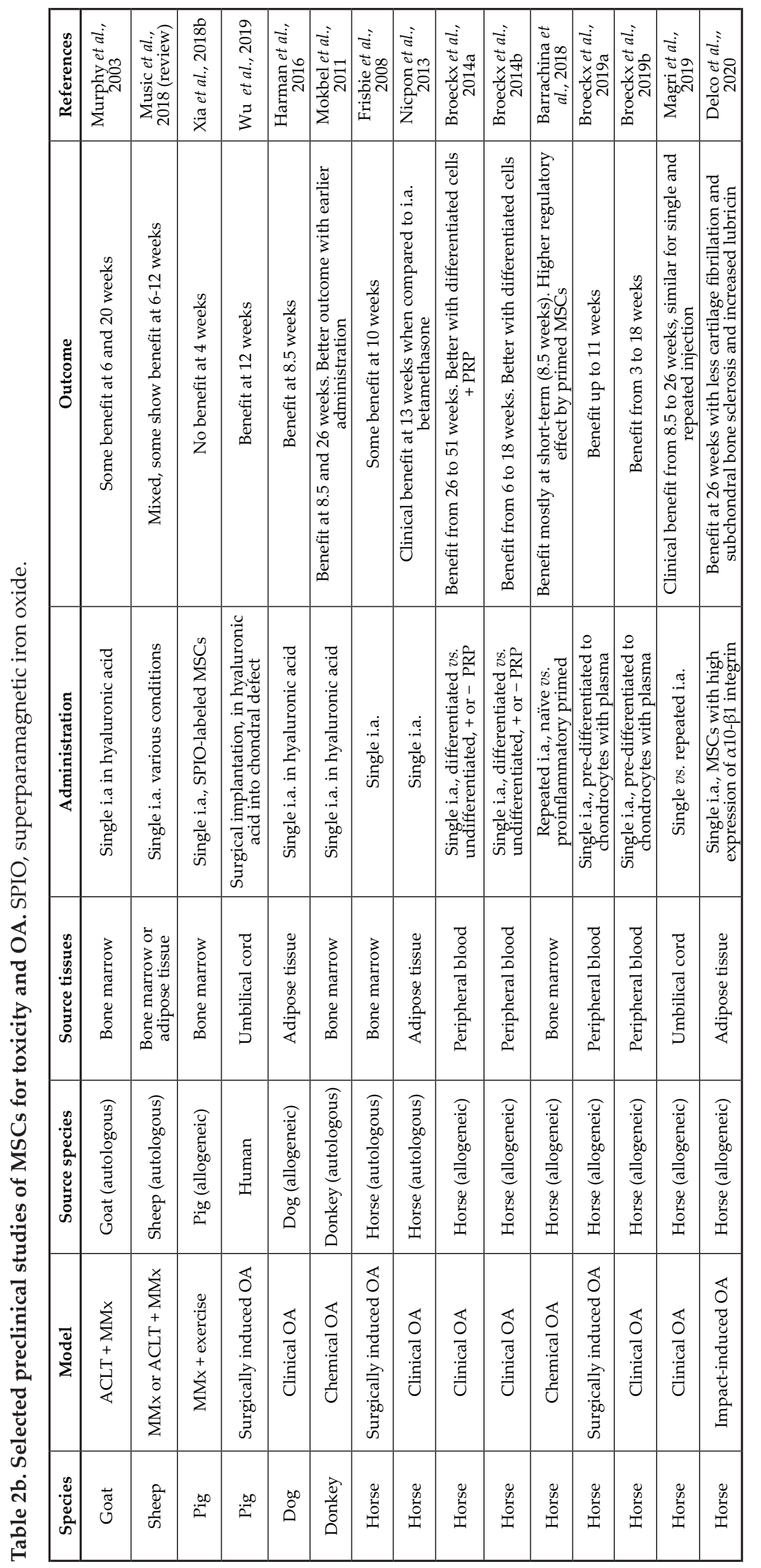

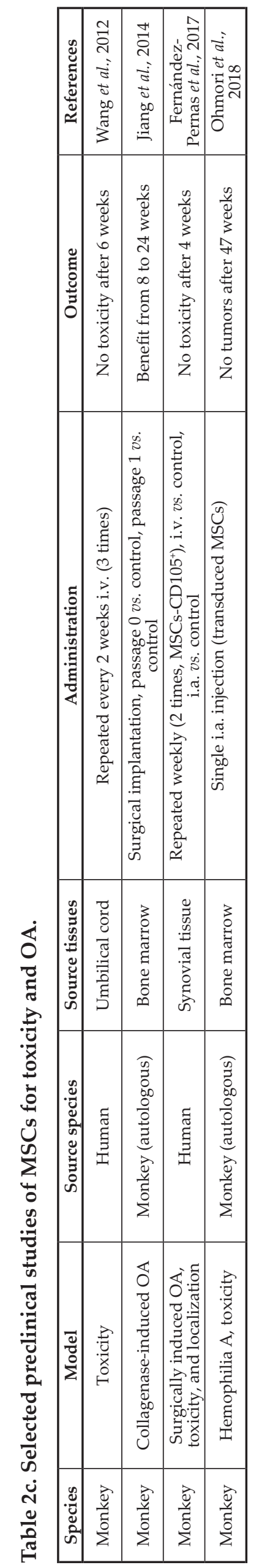




\begin{tabular}{|c|c|c|c|c|c|c|c|c|c|c|}
\hline \multirow[b]{2}{*}{ 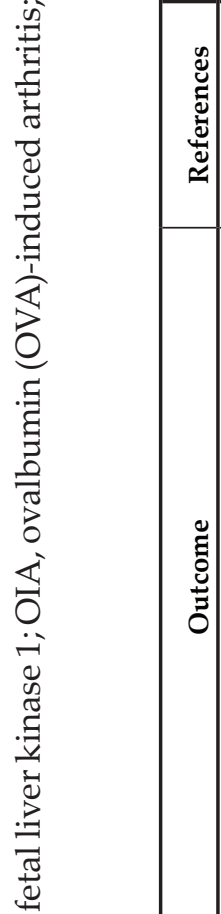 } & 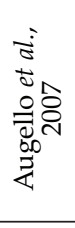 & 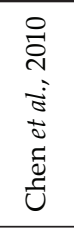 & 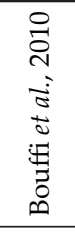 & 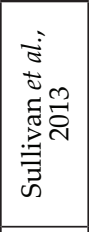 & 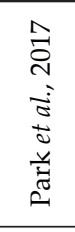 & 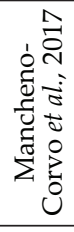 & 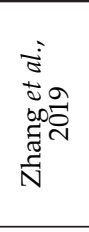 & 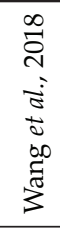 & 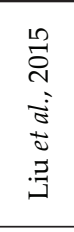 & 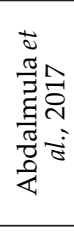 \\
\hline & 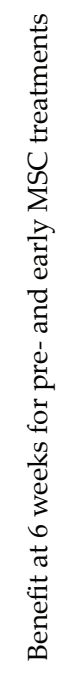 & 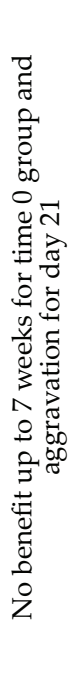 & 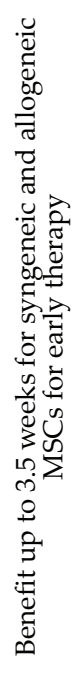 & 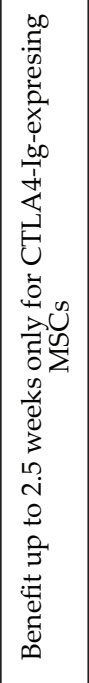 & 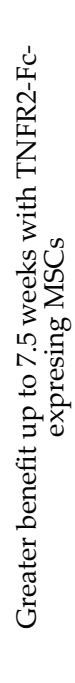 & 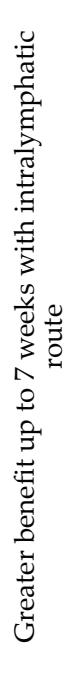 & 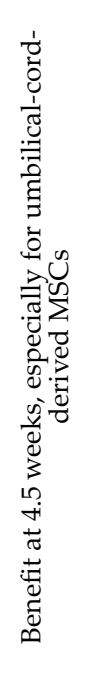 & 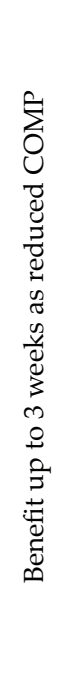 & 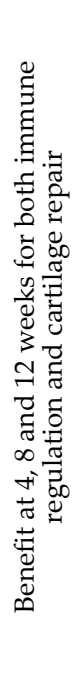 & 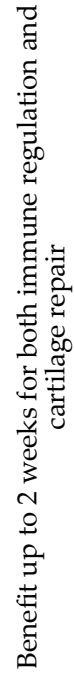 \\
\hline 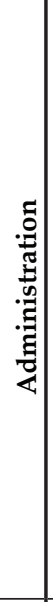 & 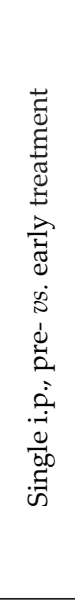 & 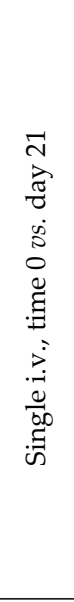 & 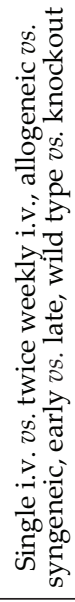 & 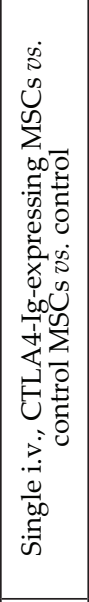 & 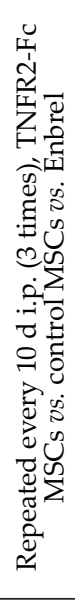 & 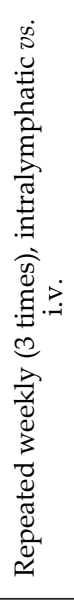 & 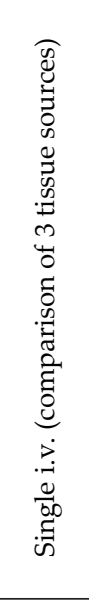 & 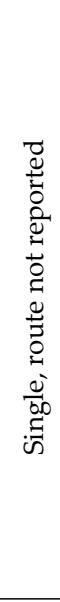 & 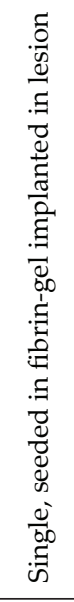 & 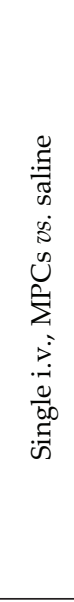 \\
\hline 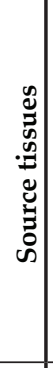 & 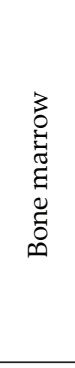 & 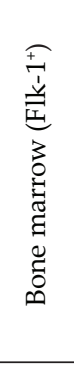 & 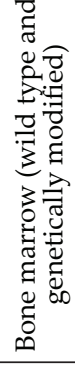 & 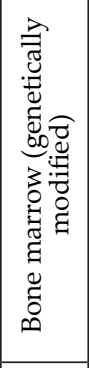 & 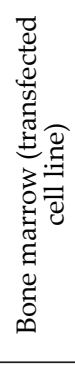 & 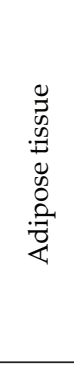 & 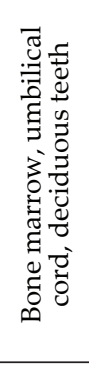 & 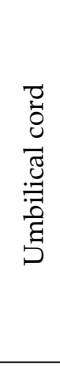 & 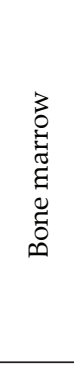 & 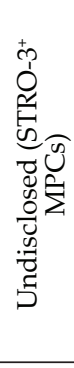 \\
\hline 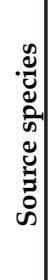 & 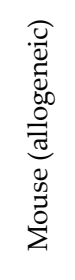 & 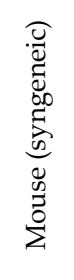 & 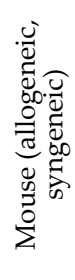 & 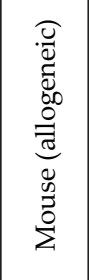 & 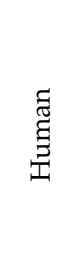 & 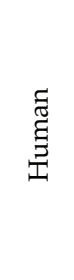 & 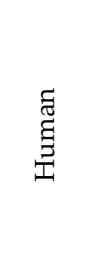 & 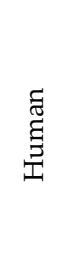 & 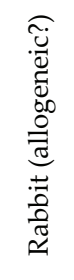 & 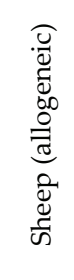 \\
\hline $\begin{array}{l}\overline{\mathrm{d}} \\
\mathrm{a} \\
\overline{\mathrm{a}}\end{array}$ & $\mathbb{U}$ & $\overleftrightarrow{U}$ & $\overleftrightarrow{U}$ & $\overleftrightarrow{U}$ & 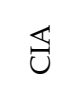 & $\overleftrightarrow{d}$ & $\overleftrightarrow{U}$ & $\mathbb{U}$ & 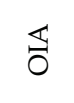 & $\mathbb{U}$ \\
\hline $\begin{array}{l}\text { : } \\
\text { ⿹े̆ } \\
\text { के }\end{array}$ & $\begin{array}{l}\mathscr{m}^{\circ} \\
\stackrel{0}{2} \\
\Sigma\end{array}$ & 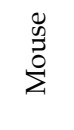 & $\begin{array}{l}\tilde{y} \\
\overline{0} \\
\bar{z}\end{array}$ & $\begin{array}{l}\tilde{y} \\
\tilde{z} \\
\bar{z}\end{array}$ & 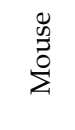 & $\begin{array}{l}0 \\
0 \\
\bar{z} \\
\Sigma\end{array}$ & 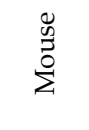 & 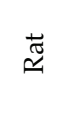 & 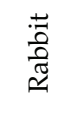 & $\begin{array}{l}\text { ष्ँ } \\
\text { ऍँ }\end{array}$ \\
\hline
\end{tabular}




\section{Preclinical studies assessing the repair of traumatic cartilage defects by MSCs}

Most studies of defect cartilage repair are focused on assessing benefit by quantity and quality determinations of the generated tissue (Table 1). Therefore, these studies assess the chondrogenic effects, either direct or indirect, of MSCs in small- and large-animal models.

Rats and rabbits have both been used for studying the use of MSCs for treating different cartilage defects. Particularly, rats have been used as models for the repair of osteochondral and meniscal defects (Table 1). However, only Okuno et al. (2014) have studied the use of allogeneic rat MSCs for these models, pointing out a constraint of this research line. Notably, most of these rat studies assess the effect of human MSCs (Horie et al., 2012; Kohli et al., 2019; Park et al., 2019), and a few use syngeneic rat MSCs (Yamaguchi et al., 2016; Yin et al., 2016). Interestingly, syngeneic and minor-mismatched synovial MSCs promote better repair than MSCs with a major mismatch in a rat model of meniscus regeneration (Okuno et al., 2014). Although MSC administration is always directed to the joint for this set of studies, there are relevant variations in form, as some involve single i.a. injections, others the combination with hydrogels, and still others use MSC aggregates with or without biomaterials. The outcomes are also diverse in terms of generation of hyaline cartilage (Kohli et al., 2019; Park et al., 2019), but most report some degree of improvement (Horie et al., 2012; Park et al., 2019; Yamaguchi et al., 2016; Yin et al., 2016). In fact, Park et al. (2019) have found that undifferentiated MSCs produce better cartilage than chondrogenic-pre-differentiated MSCs. Nevertheless, a direct comparison of chondrocytes and MSCs in their capacity to generate hyaline cartilage in an osteochondral defect in rat shows the superiority of chondrocytes (Dahlin et al., 2014). There is some controversy on this topic, but these findings are in keeping with the current clinical situation (Kwon et al., 2019). Furthermore, isolated and cultured chondrocytes also display immunoregulatory capabilities (Lohan et al., 2016). With these considerations, cellular therapies for cartilage repair based on the implantation of genetically modified porcine chondrocytes (potentially available in large amounts) are also in development (Costa et al., 2003; Marquina et al., 2017; Sommaggio et al., 2016). The interest in MSCs mainly relies on exploiting their immunoregulatory activity in combinatorial approaches with xenogeneic chondrocytes. Unmodified porcine chondrocytes injected into the rat joint trigger xenogeneic cellular and humoral immune responses. Accordingly, the immune effect of systemic administration of allogeneic MSCs (derived from bone marrow) has been initially assessed in this rat model (Marquina et al., 2017). No antibody response against MSCs was found after a single MSC injection, but the regime used did not reduce the immune response against non-transgenic chondrocytes (Marquina et al., 2017). In fact, i.v. administration of MSCs one week prior to chondrocyte i.a. injection was proinflammatory and enhanced the xenogeneic immune response. Thus, further studies are needed to assess additional protocols if MSC-mediated protective conditions are sought. Notably, this is still a requirement when the goal is cartilage regeneration.

There is a very large body of work on studying repair of cartilage defects of the joint in rabbits, also involving various models (chondral and osteochondral defects, meniscal lesions), scaffolds, and types of MSCs (Table 1). Most of these studies use rabbit MSCs from the same strain/breed and should be considered to be syngeneic or possibly with minor mismatches depending on the level of inbreeding (Cipriani et al., 2019; Guo et al., 2018; Shimomura et al., 2014; Shimomura et al., 2019; Tatebe et al., 2005; Xia et al., 2018). Overall, this intense research, with a focus on structural reconstruction, has produced encouraging results through multiple strategies based on advanced scaffolds. Likewise, the few studies testing human MSCs in rabbit models also reported good outcomes (Jang et al., 2014; Pescador et al., 2017). Notably, recent work indicates that MSCs could be better for regenerating bone than cartilage in an osteochondral defect (Cipriani et al., 2019), encouraging the development of bilayer systems. Furthermore, the generation of high-quality hyaline cartilage with zonal distributions has been recently described using procedures based on this type of approach. Particularly, Wu et al. (2020) observed that implantation of nanopatterned differentiated MSCs within a stratified bilayered hydrogel construct improved the repair quality of cartilage defects as determined by histological scoring, mechanical properties, and polarized microscopy analyses.

The use of allogeneic MSCs for this type of studies is infrequent. Nevertheless, Mahmoud et al. (2018) have recently shown that rabbit allogeneic MSCs are equally effective when compared to autologous MSCs in repairing an osteochondral defect. Furthermore, the study demonstrated that co-culturing different types of MSCs leads to the generation of betterquality hyaline cartilage and subchondral-bone repair (Mahmoud et al., 2019a). Even so, caution should be taken regarding the translation of these results into the clinical setting as allogenicity may have a higher impact in humans. Interestingly, the immunological properties of allogeneic MSCs are probably influenced by the type of scaffold used (Yuan et al., 2011) and this is an additional factor that should be considered for future studies to ensure proper development for clinical trials. Overall, further studies in high-valued preclinical animal models may be advised to validate those results and progress towards clinical applications, particularly for allogeneic cell-based products.

The efficacy of MSCs for the repair of articular chondral defects in large-animal studies has 
been mainly assessed in sheep using autologous cells, leading to variable outcomes (Table 1). The approaches tested comprise either i.a. injection of MSCs or MSCs combined with hydrogels or scaffolds placed into the generated defect (Music et al., 2018). In general, more work is needed to establish the most favorable conditions that lead to hyaline cartilage regeneration in this species. Interestingly, GuillénGarcía et al. (2014) have shown in the ovine model that autologous chondrocytes seeded at high density lead to a better cartilage repair than using the same amount of autologous MSCs. Autologous MSCs have also been investigated for the treatment of focal chondral defects in horses (Table 1). These have been conducted almost exclusively in combination with different types of scaffolds (Gonzalez-Fernandez et al., 2016; Goodrich et al., 2016; Seo et al., 2013; Wilke et al., 2007) or as a complementary treatment after surgery (Ferris et al., 2014; McIlwraith et al., 2011). Since the present review seeks to emphasize the advances been made with allogeneic cells, these studies will not be discussed extensively. Nevertheless, it is worth mentioning that promising results have been observed, even though the heterogeneity in scaffold materials and study designs hampers extracting definitive conclusions (Colbath et al., 2017a).

Despite promising results, the integration of repaired cartilage with the surrounding native cartilage remains a major challenge for tissueengineering strategies of cartilage repair. In this regard, an independent investigation focused on the incorporation of MSCs into gels to improve the integration and repair of cartilage defects using a cynomolgus macaque model with a fullthickness cartilage defect. The transplantation of autologous MSCs in a collagen gel produced a better-quality cartilage, with a regular surface and seamless integration with neighboring native cartilage, relative to using gel alone (Araki et al., 2015). More recently, Kondo et al. (2017) investigated whether transplantation of aggregates of autologous synovial MSCs promotes meniscal regeneration in aged primates, as the anatomy and biological properties of the meniscus depend on animal species. They concluded that transplantation of aggregates of autologous synovial MSCs promotes meniscus regeneration and delays progression of articular cartilage degeneration in aged primates. This work constitutes the first report dealing with meniscus regeneration in primates (Kondo et al., 2017). Unfortunately, no studies using allogeneic MSCs from non-human primates are available for comparison.

The chondrogenic potential of allogeneic MSCs obtained from large animals has been mainly studied in pig models of articular cartilage repair (Table 1) (Fisher 2016; Hatsushika et al., 2014; Nakamura et al., 2012). Although there are also variations in the degree of success, the best results have been obtained using synovial MSCs for either filling of cartilage defects (Nakamura et al., 2012) or meniscus regeneration through repeated i.a. injections (Hatsushika et al., 2014). Regarding the use of allogeneic MSCs for cartilage regeneration in sheep, this approach has been only used for the repair of lumbar intervertebral discs, with encouraging results (Freeman et al., 2016; Oheme et al., 2016). Occasionally, human MSCs have been used in these large-animal models. As in small-animal models, the fact that these cells are xenogeneic for these combinations did not seem to impact negatively upon efficacy (Hopper et al., 2015; Li et al., 2009b). Thus, these preclinical models seem appropriate under the current level of knowledge for the validation and safety analyses of human MSC preparations intended for clinical trials. Notably, no comparative studies are available that establish whether autologous MSCs provide superior benefit relative to allogeneic and human MSCs in these largeanimal models.

In summary, the use of allogeneic MSCs is gaining attention, but it is still rarely utilized in preclinical studies of osteochondral repair and especially in large-animal models. Accordingly, the implications of their use relative to autologous MSCs are not yet well established. Furthermore, it is still likely that chondrocytes represent a better cell type for the generation of high-quality hyaline cartilage. Thus, strategies that combine MSCs and chondrocytes (Nazempour and Van Wie, 2016) are also of great interest.

\section{Preclinical studies assessing MSC-based therapies for $\mathrm{OA}$}

The effect of MSCs on the treatment of OA is dual, comprising both the chondrogenic and immunoregulatory activities, in accordance with the complex pathogenesis of OA. To assess their therapeutic efficacy, there is a wide variety of OA animal models generated using different species and approaches and each studying MSCs with different origins and administration regimes (Table 2). Despite their small size, mice have been used for OA modeling, mostly following the collagenaseinduced OA model (Table 2). In keeping with the encouraging results obtained in this model, the efforts have been mostly focused on studying human MSCs for developing MSC-based therapies based on i.a. administration (Maumus et al., 2017; Morille et al., 2016). Although the molecular mechanisms of protection are not fully elucidated, the antiinflammatory and anti-catabolic effects exerted by the MSC secretome seem to play a major role (Cosenza et al., 2017; Maumus et al., 2017). Nevertheless, these findings lead to the consideration of other related strategies for therapeutic intervention such as the direct use of exosomes and/or microparticles obtained from MSCs (Cosenza et al., 2017), or even injection of MSC secretome (Khatab et al., 2018). Regarding the use of allogeneic MSCs in this model, only one report describes the use of allogeneic MSCs (an MSC 
cell line) in an OA mouse model (Xia et al., 2015). Interestingly, it showed that i.a.-injected MSCs genetically modified to produce a TNF-blocking molecule provide further protection from disease progression (Xia et al., 2015).

Various approaches have been used for OA modeling in rats. More often, an i.a. injection of MIA has been applied to rats to induce an arthritis model - considered an OA model by most groups (although occasionally reported as an RA model). The results generated in the MIA-induced arthritis and other rat OA models (Table 2) do not always support a potential beneficial effect of MSC administration (Neybecker et al., 2018; van Buul et al., 2014). For this reason, efforts have been recently focused on improving protocols (usually i.a.-based) and efficacy through various strategies such as combination with chondrocytes (Ahmed et al., 2014), repetitive MSC administration (Ozeki et al., 2016), combination with small drugs and administration routes (Lee et al., 2018), or early intervention (Sakamoto et al., 2019). Much of this preclinical work has been conducted in a xenogeneic setting, using human MSCs for determining their therapeutic potential (Lee et al., 2018; Neybecker et al., 2018; Ozeki et al., 2016). In fact, only two teams have used allogeneic MSCs in this setting (Mei et al., 2017; Yang et al., 2015). Interestingly, a beneficial effect of a single i.a. administration of allogeneic MSCs has been reported in the rat OA model generated by ACLT (Mei et al., 2017). Overall, the information regarding the immunoregulatory properties of MSCs in this setting is very limited. Nevertheless, there are indications that benefit might be associated with diminished pro-inflammatory cytokines in the joint (Lee et al., 2018; Sakamoto et al., 2019).

Multiple teams have contributed to this research topic using different OA rabbit models and types of MSCs (including two studies with allogeneic MSCs) (Table 2) (Chiang et al., 2016; Hermeto et al., 2016; Mahmoud et al., 2019b; Saulnier et al., 2015; Singh et al., 2014), adding an additional level of complexity. Nevertheless, the i.a. route has been favored for all these studies, obtaining generally beneficial effects and preventing disease progression when optimizing the conditions of MSC usage. Thus, an early intervention with MSCs is more protective than a late MSC administration (Saulnier et al., 2015). Moreover, repeated MSC injections work better than a single MSC administration (Mahmoud et al., 2019b). Notably, allogeneic MSCs were highly efficacious, although no direct comparison with the autologous or syngeneic cells was conducted in this setting .

Regarding studies in large animals, sheep have been used as OA models for assessing MSC-based therapies as reviewed by Music et al. (2018), although with mixed results (Table 2). Likewise, no consistency has been observed for the provided benefit in the two OA studies reported in pigs (Table 2). In a well-defined OA model, Xia et al. (2018b) found no difference in BM-MSC-treated lesions relative to controls. However, it is unclear whether the labelling of the MSCs with magnetic nanoparticles affect their protective functions (Xia et al., 2018b). In contrast, Wu et al. (2019) observed improved cartilage repair at both macroscopic and histological levels after two pigs were subjected to a complex surgical intervention of cartilage injury followed by transplantation with UCMSCs in hyaluronic acid. Regarding dogs, these are also used to some degree for studying the therapeutic potential of MSCs (Harman et al., 2016). However, their relevance as a preclinical model for humans is hampered by the very different cartilage load and features and its value should be based on assessing mainly the MSC immunomodulatory effects (Table 2). Thus, no conclusions can be extracted at this stage with the limited amount of information available.

Although limited, there is a larger number of studies investigating the use of MSCs as OA treatment in horses (Table 2). However, the results are less conclusive than for cartilage repair in this species. Autologous BM-MSCs have not led to a significant improvement in a post-traumatic OA experimental model, except for a reduction in the synovial concentration of PGE2 (Frisbie et al., 2009). Nevertheless, in a chemically induced OA model, equine autologous MSCs did show beneficial effects on cartilage repair, especially in the shortterm and after early cell administration (Mokbel et al., 2011). Different results between studies may be related to the more severe inflammation in the chemically-induced model (Colbath et al., 2017a), since increased MSC effectivity under inflammatory situation has been described both in vitro and in vivo in other species (Manferdini et al., 2013; Schelbergen et al., 2014). The use of allogeneic MSCs in equine joint pathologies is increasingly being investigated, but several knowledge gaps still exist. In a model of acute synovitis, allogeneic MSCs reduced the nucleated cells and neutrophil counts in synovial fluid (Williams et al., 2016). In naturally occurring OA, allogeneic MSCs undifferentiated or chondrogenically pre-differentiated showed increased beneficial effects when combined with PRP, especially the pre-differentiated ones, both in a short (6 week) and long term (12 months) (Broeckx et al., 2014a). Promising results have also been shown by using allogeneic undifferentiated or chondrogenically induced MSCs with no other orthobiological combination, the second ones reaching higher percentage of animals returning to their previous training level. Furthermore, it is remarkable that only 3 out of 165 treated horses developed a flare reaction after i.a. administration of allogeneic cells (Broeckx et al., 2014b). Recently, the same research group conducted a randomized, double-blinded, and placebo-controlled study enrolling 75 horses with early OA to assess single administration of allogeneic chondrogenically induced MSCs. Animals receiving these MSCs showed significant clinical improvement in the short term (week 3 to 18 post-treatment) when compared to the placebo group. At longer term (1 year), a significantly larger number of horses in the 
treated group returned to their previous work level. Importantly, no relevant side effects were noticed (Broeckx et al., 2019). Similar results regarding clinical benefit were obtained in a recent study that compared single and repeated administration of allogeneic UCMSCs in naturally occurring OA (Magri et al., 2019). However, a second administration of MSCs did not provide additional benefit.

Repeated allogeneic i.a. administration in equine pathological joints has also been investigated together with the effect of priming the cells in vitro with $\mathrm{TNF} \alpha$ and IFN $\gamma$ to induce their immune regulatory potential prior to administration, a suggested strategy commented above (Barrachina et al., 2018b). Clinical and synovial inflammatory signs were reduced faster in both (unstimulated and primed) MSC-treated groups when compared to untreated controls, and repeated allogeneic administration did not produce adverse reactions. However, only animals receiving primed MSCs showed a slight and transient local inflammatory reaction after the second injection, which might have resulted from increased immunogenicity of primed cells. Both MSC-treated groups showed enhanced cartilage gross appearance at short- ( 2 months) compared to long-term ( 6 months) evaluations and histochemistry suggested delayed progression of proteoglycan loss in MSC-treated groups (Barrachina et al., 2018b). The gene expression of several markers in cartilage and synovium revealed a stronger anti-inflammatory effect of primed MSCs, especially at short term (Barrachina et al., 2018b). Similarly, xenogeneic administration of equine MSCs showed that MSCs pre-stimulated with IFN $\gamma$ elicited higher chondroprotective potential when compared to unstimulated MSCs in a mouse model of joint pathology (Maumus et al., 2016). Another xenogeneic study (horse-to-rabbit model) further highlighted the importance of an early treatment and suggested that the main target of MSCs inside the joint is the synovium, since these cells are able to diminish the expression of degradative enzymes and inflammatory mediators, thus promoting an anti-catabolic joint environment (Saulnier et al., 2015). Interestingly, a recent work with allogeneic MSCs selected for high integrin expression in order to target the cells to the cartilage lesion, also produced encouraging results in a novel post-traumatic OA model (Delco et al., 2020). In summary, the study of allogeneic MSCs in equine joints is more limited than for autologous MSCs and mostly focused on OA. Allogeneic administration appears to be safe and effective, and efficacy seems to be mainly associated with MSC immunomodulatory properties. The apparently short effect of allogeneic MSC in vivo may be improved by repeating the administration, but concerns are raised regarding immune memory development.

In spite of their similarity to humans, non-human primates are complex models for assessing MSC therapeutic potential in OA (Table 2). A cynomogus monkey (Macaca fascicularis) model of OA was established by Ham et al. (2002). This animal model was initially used to assess the efficacy of different hormone treatments for OA (Ham et al., 2004; Olson et al., 2007). More recently, Fernández-Pernas et al. (2017) have demonstrated in an OA model generated by creating a lesion in the knee articular cartilage that MSCs isolated from human synovial membranes are recruited into the injured joint after intravenous injection. Furthermore, MSCs injected into the injured defect of the joint stay there, although a small percentage migrates out of the knee over time. Interestingly, native cells positive for MSC markers are also mobilized in the injured joint towards the defect independently of the MSCs injected for the lesion repair (Fernández-Pernas et al., 2017). In non-human primates, the efficacy of cartilage repair by MSCs was initially assessed in a collagenaseinduced OA model (Jiang et al., 2014). The articular cartilage lesions in cynomolgus monkey were treated locally with autologous polyclonal MSCs, a selected population of chondrogenic MSCs, or normal saline as control, and followed for 8, 16 and 24 weeks. A significant improvement was observed after evaluation of the cartilage repair by clinical, radiographic, and histological examinations in the cohorts treated with MSCs, particularly in the group treated with the selected chondrogenic clonal MSCs (Jiang et al., 2014).

There are no studies in non-human primates that assess the efficacy of well-characterized allogeneic MSCs in cartilage disease despite the high interest that may raise. Considering there are data indicating a potential effect of alloreactivity (Isakova et al., 2014), it should be taken into account in current clinical trials.

\section{Preclinical studies assessing MSC-based therapies for RA}

The development of RA therapeutic solutions based on MSCs mainly seek to take advantage of their immunoregulatory properties in accordance with the immunological origin of RA. Nevertheless, cartilage repair is still used to assess the beneficial effects. Most studies have been conducted in mice in accordance with the availability of a well-established RA mouse model, the CIA model (Augello et al., 2007; Bouffi et al., 2010; Mancheno-Corvo et al., 2017, Park et al., 2017; Zhang et al., 2019). Multiple administration routes and various sources of MSCs have been studied in this setting to develop efficacious therapeutic protocols for RA (Table 3). Systemic administration routes have been used in the CIA mouse model justified by the fact that RA is not only a disease of the joints. Early work found a protective effect when allogeneic BM-derived MSCs were administered i.p. at the time of first immunization (two immunizations are conducted in the CIA model separated by $21 \mathrm{~d}$ ) (Augello et al., 2007). However, other teams did not observe such protection and found even disease exacerbation when injected at later time points (Chen et al., 2010). Subsequent work showed that 
both syngeneic and allogeneic MSCs can display immunosuppressive effects and protect from the disease when administered in a narrow window around the time of the second immunization (Bouffi et al., 2010). Nevertheless, this effect is strain dependent as allogeneic MSCs from BALB/c mice worsen the disease outcome unless the MSCs are genetically modified with a tolerogenic molecule such as CTLA4-Ig (Sullivan et al., 2013). Human MSC preparations have also been tested in the CIA mouse model assessing multiple protocols (ManchenoCorvo et al., 2017, Park et al., 2017; Zhang et al., 2019). Intravenous injection of human MSCs from various sources (bone marrow, umbilical cord, and deciduous teeth), led to therapeutic improvement of CIA with various degrees of efficacy depending on the tissue of MSC origin (Zhang et al., 2019). Interestingly, intra-lymphatic administration of human expanded adipose-derived MSCs ameliorated CIA and promoted immune regulation (ManchenoCorvo et al., 2017). Intraperitoneal injection of human bone-marrow-derived MSCs also ameliorated CIA, especially when using MSCs genetically engineered to express the TNF-blocking drug etanercept (Park et al., 2017). Thus, multiple factors affect the outcome and point out the need for careful experimental design and controls of MSC products prior to clinical trials in RA.

MSC-based studies of RA are very infrequent in other species, especially in large animals, impairing any comparative analysis. Rats and rabbits have been used on rare occasions for RA modeling and preclinical assessment of MSC therapeutic potential (Table 3) (Liu et al., 2015; Wang et al., 2018). Interestingly, Liu et al. (2015) proposed a different approach based on implantation of MSCs seeded in a fibrin-based gel directly into subchondral defects for the repair of RA-induced cartilage injury. Their MSC product is not well defined, may be syngeneic or allogeneic, but the results are very encouraging for counteracting both inflammation and cartilage degradation. To the authors' knowledge, only one study was conducted in large animals for this purpose (Table 3) (Abdalmula et al., 2017). The work by Abdalmula et al. (2017) show good results in sheep in a well-designed preclinical study (although of short follow up). The study was conducted in preparation for clinical trials of an equivalent product of human allogeneic MPCs (as named by Mesoblast Ltd., Melbourne, Australia) currently being tested in clinical trials.

Work is thus in progress, but it is still of special interest to determine whether any or several of these animal models of OA or RA can provide relevant information that can be translated into clinical trials.

\section{Preclinical studies assessing MSC toxicity}

The study of possible MSC therapies for cartilage repair in non-human primates is highly restricted due to its ethical implications and the cost of animal housing. Only a selected number of well-designed and documented studies in these species are allowed. Non-human primates display as the main advantage for studying cartilage repair the highest similarity to humans at the molecular and physiological levels. Therefore, models such as the OA model of the cynomolgus macaque can facilitate rapid translation into clinical practice when conclusive results are obtained. However, its use for MSC research is limited to a few studies using mainly autologous cells (Kondo et al., 2017; Ohmori et al., 2018) and from humans (Fernández-Pernas et al., 2017) in a variety of models (Table 2). These models are mainly used to assess the safety, toxicity, and feasibility of their preparation as a preamble to clinical trials. Some studies do not clearly specify the exact origin of the MSCs and may be using allogeneic cells or a mixture of allogenic and autologous cells (Araki et al., 2015; Jiang et al., 2014). Long-term safety of i.a. injection of lentiviral-transduced autologous MSCs in non-human primates has been recently tested by Ohmori et al. (2018) and their migration and distribution were assessed when transplanted into the striatum of young Macaca fascicularis (Li et al., 2014). In particular, no toxicity related to stem cell transplantation was found in several non-human primate studies (Fernández-Pernas et al., 2017; Wang et al., 2012). These findings support the use of animals genetically close to humans to conduct valuable preclinical studies for the development of MSC-based therapies for cartilage repair.

\section{Concluding remarks}

The preclinical studies currently available show a potential therapeutic benefit of MSCs for cartilage repair. However, it is clear from both small and large animal models that not all protocols and conditions lead to amelioration of disease or repair. Therefore, it is crucial to optimize the cellular product (tissue source, autologous or allogeneic, live cell, or exosomes) and administration regimes (dose, frequency, route) to achieve therapeutic efficacy. Much of this information is being obtained directly in clinical trials, but a balance is necessary to avoid excessive risk and cost. To this end, rigorous studies that compare various options in relevant and wellestablished animal models are still needed to help set up the bases for additional clinical studies. With a good understanding of the limitations of the various animal models, careful consideration and design of future clinical trials will certainly profit from this type of information. Furthermore, it is also critical determining the goals and benefits that are provided by MSC treatment (cartilage repair versus immune regulation) for each targeted indication and its comparison to other approaches being currently investigated. 
Numerous preclinical studies are based on xenogeneic combinations that assess human MSCs in mice, rats, rabbits, or even occasionally in large animals. Caution should be taken when trying to translate these studies for the development of therapeutic solutions based on an allogeneic MSC product. Such results are indicative of potential safety and efficacy of a product in development but are not acceptable for modeling potential xenogeneic clinical applications (therapies based on the use of pig or bovine MSCs for human patients). Neither could be used to provide accurate information regarding the allogenicity of the cellular product as this could only be attained in humans or in co-culture assays with human immune cells. Experiments with allogeneic MSCs are scarce in small animal and ovine models but are gaining attention in recent years as the field progresses towards the clinic. Thus, more studies are needed that directly establish the equivalency or superiority of this type of MSCs when compared to autologous cells.

In summary, major advances have been made at the preclinical level and more studies are expected that will need to be validated and later assayed in clinical trials to confirm its medical utility. The use of large-animal models could be key to attain this goal. In parallel, other strategies are emerging that offer also great potential and may become complementary to the MSC-based therapies such as biomaterialguided delivery of gene vectors (Cucchiarini and Madry, 2019) and combinatorial strategies of MSCs with chondrocytes (Marquina et al., 2017; Nazempour and Van Wie, 2016). After all this vast effort on the study of MSC-based therapies and the limited clinical application currently attained based on efficacy, it is justified to set specific goals and program research projects that provide clarity on the real therapeutic potential of this technology in articular cartilage repair.

\section{Acknowledgements}

This work was funded by Ministerio de Ciencia e Innovación - Instituto de Salud Carlos III (ISCiii) (PI11/02179 granted to CC and PI11/02799 granted to MCA), the SLT002/16/00445 grant to CC funded by the Department of Health of the Generalitat de Catalunya, Ministerio de Economía y Competitividad-ISCiii (PI15/00181 granted to CC), Ministerio de Industria, Economía y Competitividad (AGL2017-84411-P granted to CR), and co-funded by FEDER funds - a way to build Europe. IDIBELL benefits from the support of CERCA. P.F.-P. benefited from a twomonth fellowship from CIBER-BBN-ISCiii.

\section{References}

Abdalmula A, Dooley LM, Kaufman C, Washington EA, House JV, Blacklaws BA, Ghosh P, Itescu S, Bailey SR, Kimpton WG (2017) Immunoselected STRO-3+ mesenchymal precursor cells reduce inflammation and improve clinical outcomes in a large animal model of monoarthritis. Stem Cell Res Ther 8: 22. DOI: 10.1186/s13287-016-0460-7.

Ankrum JA, Ong JF, Karp JM (2014) Mesenchymal stem cells: immune evasive, not immune privileged. Nature Biotech 32: 252-260.

Afizah H, Hui JH (2016) Mesenchymal stem cell therapy for osteoarthritis. J Clin Orthop Trauma 7: 177-182.

Ahmed MR, Mehmood A, Bhatti FU, Khan SN, Riazuddin S (2014) Combination of ADMSCs and chondrocytes reduces hypertrophy and improves the functional properties of osteoarthritic cartilage. Osteoarthritis Cartilage 22: 1894-1901.

Alfredson H, Lorentzon R (1999) Superior results with continuous passive motion compared to active motion after periosteal transplantation. A retrospective study of human patella cartilage defect treatment. Knee Surg Sports Traumatol Arthrosc 7: 232-238.

Almeida M, O'Brien CA (2013) Basic biology of skeletal aging: role of stress response pathways. Journals Gerontol Ser A Biol Sci Med Sci 68: 11971208.

Araki S, Imai S, Ishigaki H, Mimura T, Nishizawa K, Ueba H, Kumagai K, Kubo M, Mori K, Ogasawara K, Matsusue Y (2015) Improved quality of cartilage repair by bone marrow mesenchymal stem cells for treatment of an osteochondral defect in a cynomolgus macaque model. Acta Orthop 86: 119-126.

Ardanaz N, Vazquez FJ, Romero A, Remacha AR, Barrachina L, Sanz A, Ranera B, Vitoria A, Albareda J, Prades M, Zaragoza P, Martín-Burriel I, Rodellar C (2016) Inflammatory response to the administration of mesenchymal stem cells in an equine experimental model: effect of autologous, and single and repeat doses of pooled allogeneic cells in healthy joints. BMC Vet Res 12: 65. DOI: 10.1186/s12917-016-0692-x.

Augello A, Tasso R, Negrini SM, Cancedda R, Pennesi G (2007) Cell therapy using allogeneic bone marrow mesenchymal stem cells prevents tissue damage in collagen-induced arthritis. Arthritis Rheum 56: 1175-1186.

Barrachina L, Remacha AR, Romero A, Vazquez FJ, Albareda J, Prades M, Ranera B, Zaragoza P, Martin-Burriel I, Rodellar C (2016) Effect of inflammatory environment on equine bone marrow derived mesenchymal stem cells immunogenicity and immunomodulatory properties. Vet Immunol Immunopathol 171: 57-65.

Barrachina L, Remacha AR, Romero A, Vazquez FJ, Albareda J, Prades M, Gosalvez J, Roy R, Zaragoza P, Martin-Burriel I Rodellar C (2017) Priming equine bone marrow-derived mesenchymal stem cells with proinflammatory cytokines: implications in 
immunomodulation-immunogenicity balance, cell viability, and differentiation potential. Stem Cells Dev 26: 15-24.

Barrachina L, Remacha AR, Romero A, Zaragoza P, Vazquez FJ, Rodellar C (2018a) Differentiation of equine bone marrow derived mesenchymal stem cells increases the expression of immunogenic genes. Vet Immunol Immunopathol 200: 1-6.

Barrachina L, Remacha AR, Romero A, Vitoria A, Albareda J, Prades M, Roca M, Zaragoza P, Vazquez FJ, Rodellar C (2018b) Assessment of effectiveness and safety of repeat administration of proinflammatory primed allogeneic mesenchymal stem cells in an equine model of chemically induced osteoarthritis. BMC Vet Res 14: 241. DOI: 10.1186/s12917-018-1556-3.

Barrachina L, Cequier A, Romero A, Vitoria A, Zaragoza P, Vázquez FJ, Rodellar C (2020) Allo-antibody production after intraarticular administration of mesenchymal stem cells (MSCs) in an equine osteoarthritis model: effect of repeated administration, MSC inflammatory stimulation, and equine leukocyte antigen (ELA) compatibility. Stem Cell Res Ther 11: 52. DOI: 10.1186/s13287-020-1571-8.

Barry F, Murphy M (2013) Mesenchymal stem cells in joint disease and repair. Nat Rev Rheumatol 9: 584-594.

Beggs KJ, Lyubimov A, Borneman JN, Bartholomew A, Moseley A, Dodds R, Archambault MP, Smith AK, McIntosh KR (2006) Immunologic consequences of multiple, high-dose administration of allogeneic mesenchymal stem cells to baboons. Cell Transplant 15: 711-721.

Berglund AK, Schnabel LV (2017) Allogeneic major histocompatibility complex-mismatched equine bone marrow-derived mesenchymal stem cells are targeted for death by cytotoxic anti-major histocompatibility complex antibodies. Equine Vet J 49: 539-544.

Blazquez-Martinez A, Chiesa M, Arnalich F, Fernandez-Delgado J, Nistal M, De Miguel MP (2014) C-Kit identifies a subpopulation of mesenchymal stem cells in adipose tissue with higher telomerase expression and differentiation potential. Differentiation 87: 147-160.

Bouffi C, Bony C, Courties G, Jorgensen C, Noël D (2010) IL-6-dependent PGE2 secretion by mesenchymal stem cells inhibits local inflammation in experimental arthritis. PLoS One 5: e14247. DOI: 10.1371/journal.pone.0014247

Broeckx S, Zimmerman M, Crocetti S, Suls M, Marien T, Ferguson SJ, Chiers K, Duchateau L, Franco-Obregon A, Wuertz K, Spaas JH (2014a) Regenerative therapies for equine degenerative joint disease: a preliminary study. PloS One 9: e85917. DOI: 10.1371/journal.pone.0085917.

Broeckx S, Suls M, Beerts C, Vandenberghe A, Seys B, Wuertz-Kozak K, Duchateau L, Spaas JH (2014b) Allogenic mesenchymal stem cells as a treatment for equine degenerative joint disease: a pilot study. Curr Stem Cell Res Ther 9: 497-503.
Broeckx SY, Seys B, Suls M, Vandenberghe A, Marien T, Adriaensen E, Declercq J, Van Hecke L, Braun G, Hellmann K, Spaas JH (2019) Equine allogeneic chondrogenic induced mesenchymal stem cells are an effective treatment for degenerative joint disease in horses. Stem Cells Dev 28: 410-422.

Carrade DD, Owens SD, Galuppo LD, Vidal MA, Ferraro GL, Librach F, Buerchler S, Friedman MS, Walker NJ, Borjesson DL (2011) Clinicopathologic findings following intra-articular injection of autologous and allogeneic placentally derived equine mesenchymal stem cells in horses. Cytotherapy 13: 419-430.

Carrade DD, Lame MW, Kent MS, Clark KC, Walker NJ, Borjesson DL (2012) Comparative analysis of the immunomodulatory properties of equine adultderived mesenchymal stem cells. Cell Med 4: 1-11.

Carrade DD, Borjesson DL (2013) Immunomodulation by mesenchymal stem cells in veterinary species. Comp Med 63: 207-217.

Chan WK, AS Lau, JC Li, HK Law, YL Lau and GC Chan (2008) MHC expression kinetics and immunogenicity of mesenchymal stromal cells after short-term IFN-gamma challenge. Exp Hematol 36: 1545-1555.

Chao KC, Chao KF, Fu YS, Liu SH (2008) Isletlike clusters derived from mesenchymal stem cells in Wharton's jelly of the human umbilical cord for transplantation to control type 1 diabetes. PLoS One 3: e1451. DOI: 10.1371/journal.pone.0001451.

Chen L-B, Jiang X-B, Yang L (2004) Differentiation of rat marrow mesenchymal stem cells into pancreatic islet beta-cells. World J Gastroenterol 10: 3016-3020.

Chen FH, Tuan RS (2008) Mesenchymal stem cells in arthritic diseases. Arthritis Res Ther 10: 223. DOI: 10.1186/ar2514.

Chen B, Hu J, Liao L, Sun Z, Han Q, Song Z, Zhao RC (2010) Flk-1+ mesenchymal stem cells aggravate collagen-induced arthritis by up-regulating interleukin-6. Clin Exp Immunol 159: 292-302.

Cherian JJ, Parvizi J, Bramlet D, Lee KH, Romness DW, Mont MA (2015) Preliminary results of a phase II randomized study to determine the efficacy and safety of genetically engineered allogeneic human chondrocytes expressing TGF- $\beta 1$ in patients with grade 3 chronic degenerative joint disease of the knee. Osteoarthritis Cartilage 23: 2109-2118.

Chiang ER, Ma HL, Wang JP, Liu CL, Chen TH, Hung SC (2016) Allogeneic mesenchymal stem cells in combination with hyaluronic acid for the treatment of osteoarthritis in rabbits. PLoS One 11: e0149835. DOI: 10.1371/journal.pone.0149835.

Cipriani F, Ariño Palao B, Gonzalez de Torre I, Vega Castrillo A, Aguado Hernández HJ, Alonso Rodrigo M, Àlvarez Barcia AJ, Sanchez A, García Diaz V, Lopez Peña M, Rodriguez-Cabello JC (2019) An elastin-like recombinamer-based bioactive hydrogel embedded with mesenchymal stromal cells as an injectable scaffold for osteochondral repair. Regen Biomater 6: 335-347. 
Colbath AC, Frisbie DD, Dow SW, Kisiday JD, McIlwraith CW, Goodrich LR (2017a) Equine models for the investigation of mesenchymal stem cell therapies in orthopedic disease. Oper Techn in Sport Med 25: 41-49.

Colbath AC, Dow SW, Phillips JN, McIlwraith CW, Goodrich LR (2017b) Autologous and allogeneic equine mesenchymal stem cells exhibit equivalent immunomodulatory properties in vitro. Stem Cells Dev 26: 503-511.

Contador D, Ezquer F, Espinosa M, ArangoRodriguez M, Puebla C, Sobrevia L, Conget P (2015) Dexamethasone and rosiglitazone are sufficient and necessary for producing functional adipocytes from mesenchymal stem cells. Exp Biol Med (Maywood) 240: 1235-1246.

Cosenza S, Ruiz M, Toupet K, Jorgensen C, Noël D (2017) Mesenchymal stem cells derived exosomes and microparticles protect cartilage and bone from degradation in osteoarthritis. Sci Rep 7: 16214. DOI: 10.1038/s41598-017-15376-8.

Costa C, Brokaw JL, Wang Y, Fodor WL (2003) Delayed rejection of porcine cartilage is averted by transgenic expression of alpha1,2-fucosyltransferase. FASEB J 17: 109-111.

Cucchiarini M, Madry H (2019) Biomaterialguided delivery of gene vectors for targeted articular cartilage repair. Nat Rev Rheumatol 15: 18-29.

Cuerquis J, Romieu-Mourez R, Francois M, Routy JP, Young YK, Zhao J, Eliopoulos N (2014) Human mesenchymal stromal cells transiently increase cytokine production by activated $\mathrm{T}$ cells before suppressing T-cell proliferation: effect of interferongamma and tumor necrosis factor-alpha stimulation. Cytotherapy 16: 191-202.

Dahlin RL, Kinard LA, Lam J, Needham CJ, Lu S, Kasper FK, Mikos AG (2014) Articular chondrocytes and mesenchymal stem cells seeded on biodegradable scaffolds for the repair of cartilage in a rat osteochondral defect model. Biomaterials 35: 7460-7469.

Davies RL, Kuiper NJ (2019) Regenerative medicine: a review of the evolution of autologous chondrocyte implantation (aci) therapy. Bioengineering (Basel) 6: E22. DOI: 10.3390/bioengineering6010022.

de Miguel-Beriain I (2015) The ethics of stem cells revisited. Adv Drug Deliv Rev 82: 176-180.

De Bari C, Dell'Accio F, Tylzanowski P, Luyten FP (2001) Multipotent mesenchymal stem cells from adult human synovial membrane. Arthritis Rheum 44: 1928-1942.

De Schauwer C, Goossens K, Piepers S, Hoogewijs MK, Govaere JL, Smits K, Meyer E, Van Soom A, Van de Walle GR (2014) Characterization and profiling of immunomodulatory genes of equine mesenchymal stromal cells from non-invasive sources. Stem Cell Res Ther 5: 6. DOI: 10.1186/scrt395.

Díaz-Prado S, Muiños-López E, Hermida-Gómez T, Cicione C, Rendal-Vázquez ME, Fuentes-Boquete I, de Toro FJ, Blanco FJ (2011) Human amniotic membrane as an alternative source of stem cells for regenerative medicine. Differentiation 81: 162-171.

Dominici M, Le Blanc K, Mueller I, SlaperCortenbach I, Marini F, Krause D, Deans R, Keating A, Prockop Dj, Horwitz E (2006) Minimal criteria for defining multipotent mesenchymal stromal cells. The International Society for Cellular Therapy position statement. Cytotherapy 8: 315-317.

D'souza N, Rossignoli F, Golinelli G, Grisendi G, Spano C, Candini O, Osturu S, Catani F, Paolucci P, Horwitz EM, Dominici M (2015) Mesenchymal stem/ stromal cells as a delivery platform in cell and gene therapies. BMC Med 13: 186. DOI: 10.1186/s12916015-0426-0.

Elahi KC, Klein G, Avci-Adali M, Sievert KD, MacNeil S, Aicher WK (2016) Human mesenchymal stromal cells from different sources diverge in their expression of cell surface proteins and display distinct differentiation patterns. Stem Cells Int 2016: 5646384. DOI: $10.1155 / 2016 / 5646384$.

Fernández-Pernas P, Fafián-Labora J, LesendeRodriguez I, Mateos J, De la Fuente A, Fuentes I, De Toro Santos J, Blanco García F, Arufe MC (2016) 3, 3',5- Triiodo-L-thyronine increases in vitro chondrogenesis of mesenchymal stem cells from human umbilical cord stroma through SRC2. J Cell Biochem 117: 2097-2108.

Fernandez-Pernas P, Rodríguez-Lesende I, de la Fuente A, Mateos J, Fuentes I, De Toro J, Blanco FJ, Arufe MC (2017) CD105'-mesenchymal stem cells migrate into osteoarthritis joint: an animal model. PLoS One 12: e0188072. DOI: 10.1371/journal. pone. 0188072 .

Ferris DJ, Frisbie DD, Kisiday JD, McIlwraith CW, Hague BA, Major MD, Schneider RK, Zubrod CJ, Kawcak CE, Goodrich LR (2014) Clinical outcome after intra-articular administration of bone marrow derived mesenchymal stem cells in 33 horses with stifle injury. Vet Surg 43: 255-265.

Fisher MB, Belkin NS, Milby AH, Henning EA, Söegaard N, Kim M, Pfeifer C, Saxena V, Dodge GR, Burdick JA, Schaer TP, Steinberg DR, Mauck RL (2016) Effects of mesenchymal stem cell and growth factor delivery on cartilage repair in a mini-pig model. Cartilage 7: 174-184.

Flannery CR, Hughes CE, Schumacher BL, Tudor D, Aydelotte MB, Kuettner KE, Caterson B (1999) Articular cartilage superficial zone protein (SZP) is homologous to megakaryocyte stimulating factor precursor and Is a multifunctional proteoglycan with potential growth-promoting, cytoprotective, and lubricating properties in cartilage metabolism. Biochem Biophys Res Commun 254: 535-541.

Freeman BJ, Kuliwaba JS, Jones CF, Shu CC, Colloca CJ, Zarrinkalam MR, Mulaibrahimovic A, Gronthos S, Zannettino AC, Howell S (2016) Allogeneic mesenchymal precursor cells promote healing in postero-lateral annular lesions and improve indices of lumbar intervertebral disc degeneration in an ovine model. Spine (Phila Pa 1976) 41: 1331-1339. 
Frisbie DD, Kisiday JD, Kawcak CE, Werpy NM, Mcllwraith CW (2009) Evaluation of adiposederived stromal vascular fraction or bone marrowderived mesenchymal stem cells for treatment of osteoarthritis. J Orthop Res 27: 1675-1680.

Gonzalez-Fernandez ML, Perez-Castrillo S, Sanchez-Lazaro JA, Prieto-Fernandez JG, LopezGonzalez ME, Lobato-Perez S, Colaco BJ, Olivera ER, Villar-Suarez V (2016) Assessment of regeneration in meniscal lesions by use of mesenchymal stem cells derived from equine bone marrow and adipose tissue. Am J Vet Res 77: 779-788.

Goodrich LR, Chen AC, Werpy NM, Williams AA, Kisiday JD, Su AW, Cory E, Morley PS, McIlwraith CW, Sah RL Chu CR (2016) Addition of mesenchymal stem cells to autologous platelet-enhanced fibrin scaffolds in chondral defects: does it enhance repair? J Bone Joint Surg Am 98: 23-34.

Gong, X. Sun Z, Cui D, Xu X, Zhu H, Wang L, Qian W, Han X (2014) Isolation and characterization of lung resident mesenchymal stem cells capable of differentiating into alveolar epithelial type II cells. Cell Biol Int 38: 405-411.

Guillén-García P, Rodríguez-Iñigo E, GuillénVicente I, Caballero-Santos R, Guillén-Vicente M, Abelow S, Giménez-Gallego G, López-Alcorocho JM (2014) Increasing the dose of autologous chondrocytes improves articular cartilage repair: histological and molecular study in the sheep animal model. Cartilage 5: 114-122.

Guo W, Zheng X, Zhang W, Chen M, Wang Z, Hao C, Huang J, Yuan Z, Zhang Y, Wang M, Peng J, Wang A, Wang Y, Sui X, Xu W, Liu S, Lu S, Guo Q (2018) Mesenchymal stem cells in oriented PLGA/ACECM composite scaffolds enhance structure-specific regeneration of hyaline cartilage in a rabbit model. Stem Cells Int 2018: 6542198. DOI: 10.1155/2018/6542198.

Ham KD, Loeser RF, Lindgren BR, Carlson CS (2002) Effects of long-term estrogen replacement therapy on osteoarthritis severity in cynomolgus monkeys. Arthritis Rheum 46: 1956-1964.

Ham KD, Oegema TR, Loeser RF, Carlson CS (2004) Effects of long-term estrogen replacement therapy on articular cartilage IGFBP-2, IGFBP-3, collagen and proteoglycan levels in ovariectomized cynomolgus monkeys. Osteoarthritis Cartilage 12: 160-168.

Harman R, Carlson K, Gaynor J, Gustafson S, Dhupa S, Clement K, Hoelzler M, McCarthy T, Schwartz P, Adams C (2016) A prospective, randomized, masked, and placebo-controlled efficacy study of intraarticular allogeneic adipose stem cells for the treatment of osteoarthritis in dogs. Front Vet Sci 3: 81. DOI: 10.3389/fvets.2016.00081.

Hatsushika D, Muneta T, Nakamura T, Horie M, Koga H, Nakagawa Y, Tsuji K, Hishikawa S, Kobayashi E, Sekiya I (2014) Repetitive allogeneic intraarticular injections of synovial mesenchymal stem cells promote meniscus regeneration in a porcine massive meniscus defect model. Osteoarthritis Cartilage 22: 941-950.

Hermeto LC, DeRossi R, Oliveira RJ, Pesarini JR, Antoniolli-Silva AC, Jardim PH, Santana AE, Deffune E, Rinaldi JC, Justulin LA (2016) Effects of intra-articular injection of mesenchymal stem cells associated with platelet-rich plasma in a rabbit model of osteoarthritis. Genet Mol Res 15: gmr8569. DOI: 10.4238/gmr.15038569.

Horie M, Choi H, Lee RH, Reger RL, Ylostalo J, Muneta T, Sekiya I, Prockop DJ (2012) Intra-articular injection of human mesenchymal stem cells (MSCs) promote rat meniscal regeneration by being activated to express Indian hedgehog that enhances expression of type II collagen. Osteoarthritis Cartilage 20: 11971207.

Hopper N, Wardale J, Brooks R, Power J, Rushton N, Henson F (2015) Peripheral blood mononuclear cells enhance cartilage repair in in vivo osteochondral defect model. PLoS One 10: e0133937. DOI: 10.1371/ journal.pone.0133937.

Hunziker EB, Quinn TM, Häuselmann H-J (2002) Quantitative structural organization of normal adult human articular cartilage. Osteoarthritis Cartilage 10: 564-572.

Isakova IA, Lanclos C, Bruhn J, Kuroda MJ, Baker KC, Krishnappa V, Phinney DG (2014) Allo-reactivity of mesenchymal stem cells in rhesus macaques is dose and haplotype dependent and limits durable cell engraftment in vivo. PLoS One 9: e87238. DOI: 10.1371/journal.pone.0087238.

Iudicone $\mathrm{P}$, Fioravanti $\mathrm{D}$, Bonanno $\mathrm{G}$, Miceli M, Lavorino C, Totta P, Frati L, Nuti M, Pierelli L (2014) Pathogen-free, plasma-poor platelet lysate and expansion of human mesenchymal stem cells. J Transl Med 12: 28. DOI: 10.1186/1479-5876-12-28.

Jang KM, Lee JH, Park CM, Song HR, Wang JH (2014) Xenotransplantation of human mesenchymal stem cells for repair of osteochondral defects in rabbits using osteochondral biphasic composite constructs. Knee Surg Sports Traumatol Arthrosc 22: 1434-1444.

Jeon Y-J, Kim J, Cho JH, Chung H-M, Chae J-I (2016) Comparative analysis of human mesenchymal stem cells derived from bone marrow, placenta and adipose tissue as sources of cell therapy. J Cell Biochem 117: 1112-1125.

Jiang Y, Jahagirdar BN, Reinhardt RL, Schwartz RE, Keene CD, Ortiz-Gonzalez XR, Reyes M, Lenvik T, Lund T, Blackstad M, Du J, Aldrich S, Lisberg A, Low WC, Largaespada DA, Verfaillie CM (2002) Pluripotency of mesenchymal stem cells derived from adult marrow. Nature 418: 41-49.

Jiang L, Ma A, Song L, Hu Y, Dun H, Daloze P, Yu Y, Jiang J, Zafarullah M, Chen H (2014) Cartilage regeneration by selected chondrogenic clonal mesenchymal stem cells in the collagenase-induced monkey osteoarthritis model. J Tissue Eng Regen Med 8: 896-905.

Jin HJ, Bae YK, Kim M, Kwon SJ, Jeon HB, Choi SJ, Kim SW, Yang YS, Oh W, Chang JW (2013) 
Comparative analysis of human mesenchymal stem cells from bone marrow, adipose tissue, and umbilical cord blood as sources of cell therapy. Int J Mol Sci 14: 17986-18001.

Jones KJ, Kelley BV, Arshi A, McAllister DR, Fabricant PD (2019) Comparative effectiveness of cartilage repair with respect to the minimal clinically important difference. Am J Sports Med 47: 3284-3293.

Joswig AJ, Mitchell A, Cummings KJ, Levine GJ, Gregory CA, Smith R, 3rd, Watts AE (2017) Repeated intra-articular injection of allogeneic mesenchymal stem cells causes an adverse response compared to autologous cells in the equine model. Stem Cell Res Ther 8: 42. DOI: 10.1186/s13287-017-0503-8.

Jung Y, Bauer G, Nolta JA (2012) Concise review: induced pluripotent stem cell-derived mesenchymal stem cells: progress toward safe clinical products. Stem Cells 30: 42-47.

Karaoz E, Aksoy A, Ayhan S, Sariboyaci AE, Kaymaz F, Kasap M (2009) Characterization of mesenchymal stem cells from rat bone marrow: Ultrastructural properties, differentiation potential and immunophenotypic markers. Histochem Cell Biol 132: 533-546.

Khatab S, van Osch GJ, Kops N, BastiaansenJenniskens YM, Bos PK, Verhaar JA, Bernsen MR, van Buul GM (2018) Mesenchymal stem cell secretome reduces pain and prevents cartilage damage in a murine osteoarthritis model. Eur Cell Mater 36: 218230.

Kil K, Choi MY, Ho Park K (2016) In vitro differentiation of human Wharton's jelly-derived mesenchymal stem cells into auditory hair cells and neurons. J Int Adv Otol 12: 37-42.

Kohli N, Al-Delfi IRT, Snow M, Sakamoto T, Miyazaki T, Nakajima H, Uchida K, Johnson WEB (2019) CD271-selected mesenchymal stem cells from adipose tissue enhance cartilage repair and are less angiogenic than plastic adherent mesenchymal stem cells. Sci Rep 9: 3194. DOI: 10.1038/s41598-01939715-z.

Kondo S, Muneta T, Nakagawa Y, Koga H, Watanabe T, Tsuji K, Sotome S, Okawa A, Kiuchi S, Ono H, Mizuno M, Sekiya I (2017) Transplantation of autologous synovial mesenchymal stem cells promotes meniscus regeneration in aged primates. J Orthop Res 35: 1274-1282.

Kwon H, Brown WE, Lee CA, Wang D, Paschos N, Hu JC, Athanasiou KA (2019) Surgical and tissue engineering strategies for articular cartilage and meniscus repair. Nat Rev Rheumatol 15: 550-570.

Lee SY, Lee SH, Na HS, Kwon JY, Kim GY, Jung K, Cho KH, Kim SA, Go EJ, Park MJ, Baek JA, Choi SY, Jhun J, Park SH, Kim SJ, Cho ML (2018) The therapeutic effect of STAT3 signaling-suppressed MSC on pain and articular cartilage damage in a rat model of monosodium iodoacetate-induced osteoarthritis. Front Immunol 9: 2881. DOI: 10.3389/ fimmu.2018.02881.
Li G, Zhang XA, Wang H, Wang X, Meng CL, Chan CY, Yew DT, Tsang KS, Li K, Tsai SN, Ngai SM, Han ZC, Lin MC, He ML, Kung HF (2009) Comparative proteomic analysis of mesenchymal stem cells derived from human bone marrow, umbilical cord, and placenta: implication in the migration. Proteomics 9: 20-30.

Li WJ, Chiang H, Kuo TF, Lee HS, Jiang CC, Tuan RS (2009b) Evaluation of articular cartilage repair using biodegradable nanofibrous scaffolds in a swine model: a pilot study. J Tissue Eng Regen Med 3: 1-10.

Li J, Zhu H, Chen Y, Deng W, Li Q, Lu S, Xu Y, Huang L, Ma C, Zhao C, Wang R, Qin C (2014) The distribution of transplanted human mesenchymal stem cells in the CNS of young Macaca fascicularis. Brain Res 1579: 1-9.

Liang M-S, Koobatian M, Lei P, Swartz DD, Andreadis ST (2013) Differential and synergistic effects of mechanical stimulation and growth factor presentation on vascular wall function. Biomaterials 34: 7281-7291.

Liu H, Ding J, Wang C, Wang J, Wang Y, Yang M, Jia Y, Zhang Y, Chang F, Li R, Chen X (2015) Intraarticular transplantation of allogeneic BMMSCs rehabilitates cartilage injury of antigen-induced arthritis. Tissue Eng Part A 21: 2733-2743.

Liu L, Wong CW, Han M, Farhoodi HP, Liu G, Liu Y, Liao W, Zhao W (2019) Meta-analysis of preclinical studies of mesenchymal stromal cells to treat rheumatoid arthritis. EbioMedicine 47: 563-577.

Lohan P, Coleman CM, Murphy JM, Griffin MD, Ritter T, Ryan AE (2014) Changes in immunological profile of allogeneic mesenchymal stem cells after differentiation: should we be concerned? Stem Cell Res Ther 5: 99. DOI: 10.1186/scrt488.

Lohan P, Treacy O, Lynch K, Barry F, Murphy M, Griffin MD, Ritter T, Ryan AE (2016) Culture expanded primary chondrocytes have potent immunomodulatory properties and do not induce an allogeneic immune response. Osteoarthritis Cartilage 24: 521-533.

Lo Monaco M, Merckx G, Ratajczak J, Gervois P, Hilkens P, Clegg P, Bronckaers A, Vandeweerd JM, Lambrichts I (2018) Stem cells for cartilage repair: preclinical studies and insights in translational animal models and outcome measures. Stem Cells Int 2018: 9079538. DOI: 10.1155/2018/9079538.

Lopes EBP, Filiberti A, Husain SA, Humphrey MB (2017) Immune contributions to osteoarthritis. Curr Osteoporos Rep 15: 593-600.

Lorentzon R, Alfredson H, Hildingsson C (1998) Treatment of deep cartilage defects of the patella with periosteal transplantation. Knee Surg Sports Traumatol Arthrosc 6: 202-208.

Magri C, Schramme M, Febre M, Cauvin E, Labadie F, Saulnier N, François I, Lechartier A, Aebischer D, Moncelet AS, Maddens S (2019) Comparison of efficacy and safety of single versus repeated intra-articular injection of allogeneic 
neonatal mesenchymal stem cells for treatment of osteoarthritis of the metacarpophalangeal/ metatarsophalangeal joint in horses: a clinical pilot study. PLoS One 14: e0221317. DOI: 10.1371/journal. pone.0221317.

Mahmoud EE, Tanaka Y, Kamei N, Harada Y, Ohdan H, Adachi N, Ochi M (2018) Monitoring immune response after allogeneic transplantation of mesenchymal stem cells for osteochondral repair. J Tissue Eng Regen Med 12: e275-e286.

Mahmoud EE, Adachi N, Mawas AS, Gaarour OS, Ochi M (2019a) Coculturing of mesenchymal stem cells of different sources improved regenerative capability of osteochondral defect in the mature rabbit: an in vivo study. J Orthop Surg (Hong Kong) 27: 2309499019839850 . DOI: 10.1177/2309499019839850.

Mahmoud EE, Adachi N, Mawas AS, Deie M, Ochi M (2019b) Multiple intra-articular injections of allogeneic bone marrow-derived stem cells potentially improve knee lesions resulting from surgically induced osteoarthritis: an animal study. Bone Joint J 101-B: 824-831.

Mancheño-Corvo P, Lopez-Santalla M, Menta R, DelaRosa O, Mulero F, Del Rio B, Ramirez C, Büscher D, Bueren JA, Lopez-Belmonte J, Dalemans W, Garin MI, Lombardo E (2017) Intralymphatic administration of adipose mesenchymal stem cells reduces the severity of collagen-induced experimental arthritis. Front Immunol 8: 462. DOI: 10.3389/fimmu.2017.00462.

Manferdini C, Maumus M, Gabusi E, Piacentini A, Filardo G, Peyrafitte JA, Jorgensen C, Bourin P, Fleury-Cappellesso S, Facchini A, Noël D, Lisignoli G (2013) Adipose-derived mesenchymal stem cells exert antiinflammatory effects on chondrocytes and synoviocytes from osteoarthritis patients through prostaglandin E2. Arthritis Rheum 65: 1271-1281.

Manini I., Gulino L, Gava B, Pierantozzi E, Curina C, Rossi D, Brafa A, D'Aniello C, Sorrentino $\mathrm{V}$ (2011) Multi-potent progenitors in freshly isolated and cultured human mesenchymal stem cells: a comparison between adipose and dermal tissue. Cell Tissue Res 344: 85-95.

Marquina M, Collado JA, Pérez-Cruz M, Fernández-Pernas P, Fafián-Labora J, Blanco FJ, Máñez R, Arufe MC, Costa C (2017) Biodistribution and immunogenicity of allogeneic mesenchymal stem cells in a rat model of intraarticular chondrocyte xenotransplantation. Front Immunol 8: 1465. DOI: 10.3389/fimmu.2017.01465.

Marycz K, Kornicka K, Basinska K, Czyrek A (2016) Equine metabolic syndrome affects viability, senescence, and stress factors of equine adiposederived mesenchymal stromal stem cells: new insight into eqASCs isolated from EMS horses in the context of their aging. Oxid Med Cell Longev 2016: 4710326. DOI: 10.1155/2016/4710326.

Maumus M, Roussignol G, Toupet K, Penarier G, Bentz I, Teixeira S, Oustric D, Jung M, Lepage O, Steinberg R, Jorgensen C, Noel D (2016) Utility of a mouse model of osteoarthritis to demonstrate cartilage protection by IFNgamma-primed equine mesenchymal stem cells. Front Immunol 7: 392. DOI: 10.3389/fimmu.2016.00392.

Maumus M, Manferdini C, Toupet K, Chuchana P, Casteilla L, Gachet M, Jorgensen C, Lisignoli G, Noel D (2017) Thrombospondin-1 partly mediates the cartilage protective effect of adipose-derived mesenchymal stem cells in osteoarthritis. Front Immunol 8: 1638. DOI: 10.3389/fimmu.2017.01638.

McIlwraith CW, Frisbie DD, Rodkey WG, Kisiday JD, Werpy NM, Kawcak CE, Steadman JR (2011) Evaluation of intra-articular mesenchymal stem cells to augment healing of microfractured chondral defects. Arthroscopy 27: 1552-1561.

McCoy B, Miniaci A (2012) Osteochondral autograft transplantation/mosaicplasty. J Knee Surg 25: 99-108.

Mei L, Shen B, Ling P, Liu S, Xue J, Liu F, Shao H, Chen J, Ma A, Liu X (2017) Culture-expanded allogenic adipose tissue-derived stem cells attenuate cartilage degeneration in an experimental rat osteoarthritis model. PLoS One 12: e0176107. DOI: 10.1371/journal.pone.0176107.

Mokbel AN, El Tookhy OS, Shamaa AA, Rashed LA, Sabry D, El Sayed AM (2011) Homing and reparative effect of intra-articular injection of autologus mesenchymal stem cells in osteoarthritic animal model. BMC Musculoskelet Disord 12: 259. DOI: 10.1186/1471-2474-12-259.

Morille M, Toupet K, Montero-Menei CN, Jorgensen C, Noël D (2016) PLGA-based microcarriers induce mesenchymal stem cell chondrogenesis and stimulate cartilage repair in osteoarthritis. Biomaterials 88: 60-69.

Moseley JB, O’Malley K, Petersen NJ, Menke TJ, Brody BA, Kuykendall DH, Hollingsworth JC, Ashton CM, Wray NP (2002) A controlled trial of arthroscopic surgery for osteoarthritis of the knee. N Engl J Med 347: 81-88.

Murphy JM, Fink DJ, Hunziker EB, Barry FP (2003) Stem cell therapy in a caprine model of osteoarthritis. Arthritis Rheum 48: 3464-3474.

Music E, Futrega K, Doran MR (2018) Sheep as a model for evaluating mesenchymal stem/stromal cell (MSC)-based chondral defect repair. Osteoarthritis Cartilage 26: 730-740.

Nakamura T, Sekiya I, Muneta T, Hatsushika D, Horie M, Tsuji K, Kawarasaki T, Watanabe A, Hishikawa S, Fujimoto Y, Tanaka H, Kobayashi E (2012) Arthroscopic, histological and MRI analyses of cartilage repair after a minimally invasive method of transplantation of allogeneic synovial meenchymal stromal cells into cartilage defects in pigs. Cytotherapy 14: 327-338.

Nazempour A, Van Wie BJ (2016) Chondrocytes, mesenchymal stem cells, and their combination in articular cartilage regenerative medicine. Ann Biomed Eng 44: 1325-1354.

Neybecker P, Henrionnet C, Pape E, Mainard D, Galois L, Loeuille D, Gillet P, Pinzano A (2018) In vitro and in vivo potentialities for cartilage repair 
from human advanced knee osteoarthritis synovial fluid-derived mesenchymal stem cells. Stem Cell Res Ther 9: 329. DOI: 10.1186/s13287-018-1071-2.

Oehme D, Ghosh P, Goldschlager T, Itescu S, Shimon S, Wu J, McDonald C, Troupis JM, Rosenfeld JV, Jenkin G (2016) Reconstitution of degenerated ovine lumbar discs by STRO-3-positive allogeneic mesenchymal precursor cells combined with pentosan polysulfate. J Neurosurg Spine 24: 715-726.

O’Hara BP, Urban JP, Maroudas A (1990) Influence of cyclic loading on the nutrition of articular cartilage. Ann Rheum Dis 49: 536-539.

Ohmori T, Mizukami H, Katakai Y, Kawai S, Nakamura H, Inoue M, Shu T, Sugimoto H, Sakata Y (2018) Safety of intra-articular transplantation of lentivirally transduced mesenchymal stromal cells for haemophilic arthropathy in a non-human primate. Int J Hematol 108: 239-245.

Okuno M, Muneta T, Koga H, Ozeki N, Nakagawa Y, Tsuji K, Yoshiya S, Sekiya I (2014) Meniscus regeneration by syngeneic, minor mismatched, and major mismatched transplantation of synovial mesenchymal stem cells in a rat model. J Orthop Res 32: 928-936.

Olson EJ, Lindgren BR, Carlson CS (2007) Effects of long-term estrogen replacement therapy on the prevalence and area of periarticular tibial osteophytes in surgically postmenopausal cynomolgus monkeys. Bone 41: 282-289.

Ozeki N, Muneta T, Koga H, Nakagawa Y, Mizuno M, Tsuji K, Mabuchi Y, Akazawa C, Kobayashi E, Matsumoto K, Futamura K, Saito T, Sekiya I (2016) Not single but periodic injections of synovial mesenchymal stem cells maintain viable cells in knees and inhibit osteoarthritis progression in rats. Osteoarthritis Cartilage 24: 1061-1070.

Pacini S, Petrini I (2014) Are MSCs angiogenic cells? New insights on human nestin-positive bone marrow-derived multipotent cells. Cell Dev Biol 2: 1-11.

Pappa KI., Anagnou NP (2009) Novel sources of fetal stem cells: where do they fit on the developmental continuum? Regen Med 4: 423-433.

Park S, Choi Y, Jung N, Yu Y, Ryu KH, Kim HS, Jo I, Choi BO, Jung SC (2016) Myogenic differentiation potential of human tonsil-derived mesenchymal stem cells and their potential for use to promote skeletal muscle regeneration. Int J Mol Med 37: 1209-1220.

Park N, Rim YA, Jung H, Kim J, Yi H, Kim Y, Jang Y, Jung SM, Lee J, Kwok SK, Park SH, Ju JH (2017) Etanercept-synthesising mesenchymal stem cells efficiently ameliorate collagen-induced arthritis. Sci Rep 7: 39593. DOI: 10.1038/srep39593.

Park YB, Ha CW, Kim JA, Kim S, Park YG (2019) Comparison of undifferentiated versus chondrogenic predifferentiated mesenchymal stem cells derived from human umbilical cord blood for cartilage repair in a rat model. Am J Sports Med 47: 451-461.

Pelekanos RA, Sardesai VS, Futrega K, Lott WB, Kuhn M, Doran MR (2016) Isolation and expansion of mesenchymal stem/stromal cells derived from human placenta tissue. J Vis Exp 6: 54204. DOI: 10.3791/54204.

Pescador D, Ibáñez-Fonseca A, Sánchez-Guijo F, Briñón JG, Arias FJ, Muntión S, Hernández C, Girotti A, Alonso M, Del Cañizo MC, Rodríguez-Cabello JC, Blanco JF (2017) Regeneration of hyaline cartilage promoted by xenogeneic mesenchymal stromal cells embedded within elastin-like recombinamer-based bioactive hydrogels. J Mater Sci Mater Med 28: 115. DOI: 10.1007/s10856-017-5928-1.

Pezzanite LM, Fortier LA, Antczak DF, Cassano JM, Brosnahan MM, Miller D, Schnabel LV (2015) Equine allogeneic bone marrow-derived mesenchymal stromal cells elicit antibody responses in vivo. Stem Cell Res Ther 6: 54. DOI: 10.1186/s13287-015-0053-x.

Pigott JH, Ishihara A, Wellman ML, Russell DS, Bertone AL (2013a) Inflammatory effects of autologous, genetically modified autologous, allogeneic, and xenogeneic mesenchymal stem cells after intra-articular injection in horses. Vet Comp Orthop Traumatol 26: 453-460.

Pigott JH, Ishihara A, Wellman ML, Russell DS, Bertone AL (2013b) Investigation of the immune response to autologous, allogeneic, and xenogeneic mesenchymal stem cells after intra-articular injection in horses. Vet Immunol Immunopathol 156: 99-106.

Poncelet AJ, Vercruysse J, Saliez A, Gianello P (2007) Although pig allogeneic mesenchymal stem cells are not immunogenic in vitro, intracardiac injection elicits an immune response in vivo. Transplantation 83: 783-790.

Poole AR, Kojima T, Yasuda T, Mwale F, Kobayashi M, Laverty S (2001) Composition and structure of articular cartilage: a template for tissue repair. Clin Orthop Relat Res 391 Suppl: S26-33.

Ranera B, Antczak D, Miller D, Doroshenkova T, Ryan A, Mcllwraith CW, Barry F (2016) Donorderived equine mesenchymal stem cells suppress proliferation of mismatched lymphocytes. Equine Vet J 48: 253-260.

Riboh JC, Cvetanovich GL, Cole BJ, Yanke AB (2017) Comparative efficacy of cartilage repair procedures in the knee: a network meta-analysis. Knee Surg Sports Traumatol Arthrosc 25: 3786-3799.

Romero A, Barrachina L, Ranera B, Remacha AR, Moreno B, de Blas I, Sanz A, Vazquez FJ, Vitoria A, Junquera C, Zaragoza P, Rodellar C (2017) Comparison of autologous bone marrow and adipose tissue derived mesenchymal stem cells, and platelet rich plasma, for treating surgically induced lesions of the equine superficial digital flexor tendon. Vet J 224: 76-84.

Rossignoli F, Caselli A, Grisendi G, Piccinno S, Burns JS, Murgia A, Veronesi E, Loschi P, Masini C, Conte P, Paolucci P, Horwiz EM, Dominici M (2013) Isolation, characterization, and transduction of endometrial decidual tissue multipotent mesenchymal stromal/stem cells from menstrual blood. Biomed Res Int 2013: 901821. DOI: 10.1155/2013/901821.

Ruiz M, Cosenza S, Maumus M, Jorgensen C, Noël D (2016) Therapeutic application of mesenchymal 
stem cells in osteoarthritis. Expert Opin Biol Ther 16: 33-42.

Ryan AE, Lohan P, O'Flynn L, Treacy O, Chen X, Coleman C, Shaw G, Murphy M, Barry F, Griffin MD, Ritter T (2014) Chondrogenic differentiation increases antidonor immune response to allogeneic mesenchymal stem cell transplantation. Mol Ther 22: 655-667.

Sakamoto T, Miyazaki T, Watanabe S, Takahashi A, Honjoh K, Nakajima H, Oki H, Kokubo Y, Matsumine A (2019) Intraarticular injection of processed lipoaspirate cells has anti-inflammatory and analgesic effects but does not improve degenerative changes in murine monoiodoacetate-induced osteoarthritis. BMC Musculoskelet Disord 20: 335. DOI: 10.1186/ s12891-019-2710-1.

Sato M, Yamato M, Mitani G, Takagaki T, Hamahashi K, Nakamura Y, Ishihara M, Matoba R, Kobayashi H, Okano T, Mochida J, Watanabe M (2019) Combined surgery and chondrocyte cell-sheet transplantation improves clinical and structural outcomes in knee osteoarthritis. NPJ Regen Med 4: 4. DOI: 10.1038/s41536-019-0069-4.

Saulnier N, Viguier E, Perrier-Groult E, Chenu C, Pillet E, Roger T, Maddens S, Boulocher C (2015) Intra-articular administration of xenogeneic neonatal mesenchymal stromal cells early after meniscal injury down-regulates metalloproteinase gene expression in synovium and prevents cartilage degradation in a rabbit model of osteoarthritis. Osteoarthritis Cartilage 23: 122-133.

Schelbergen RF, van Dalen S, ter Huurne M, Roth J, Vogl T, Noël D, Jorgensen C, van den Berg WB, van de Loo FA, Blom AB, van Lent PL (2014) Treatment efficacy of adipose-derived stem cells in experimental osteoarthritis is driven by high synovial activation and reflected by S100A8/A9 serum levels. Osteoarthritis Cartilage 22: 1158-1166.

Schinhan M, Gruber M, Dorotka R, Pilz M, Stelzeneder D, Chiari C, Rössler N, Windhager R, Nehrer S (2013) Matrix-associated autologous chondrocyte transplantation in a compartmentalized early stage of osteoarthritis. Osteoarthritis Cartilage 21: 217-225.

Secco M, Zucconi E, Vieira NM, Fogaça LL, Cerqueira A, Carvalho MD, Jazedje T, Okamoto OK, Muotri AR, Zatz M (2008) Multipotent stem cells from umbilical cord: cord is richer than blood! Stem Cells 26: $146-150$.

Sellam J, Berenbaum F (2010) The role of synovitis in pathophysiology and clinical symptoms of osteoarthritis. Nat Rev Rheumatol 6: 625-635.

Seo JP, Tanabe T, Tsuzuki N, Haneda S, Yamada K, Furuoka H, Tabata Y, Sasaki N (2013) Effects of bilayer gelatin/beta-tricalcium phosphate sponges loaded with mesenchymal stem cells, chondrocytes, bone morphogenetic protein-2, and platelet rich plasma on osteochondral defects of the talus in horses. Res Vet Sci 95: 1210-1216.

Shanmugaraj A, Coughlin RP, Kuper GN, Ekhtiari S, Simunovic N, Musahl V, Ayeni OR (2019) Changing trends in the use of cartilage restoration techniques for the patellofemoral joint: a systematic review. Knee Surg Sports Traumatol Arthrosc. 27: 854-867.

Shi Y, Inoue H, Wu JC, Yamanaka S (2017) Induced pluripotent stem cell technology: a decade of progress. Nat Rev Drug Discov 16: 115-130.

Shimomura K, Moriguchi Y, Ando W, Nansai R, Fujie H, Hart DA, Gobbi A, Kita K, Horibe S, Shino K, Yoshikawa H, Nakamura N (2014) Osteochondral repair using a scaffold-free tissue-engineered construct derived from synovial mesenchymal stem cells and a hydroxyapatite-based artificial bone. Tissue Eng Part A 20: 2291-2304.

Shimomura K, Rothrauff BB, Hart DA, Hamamoto S, Kobayashi M, Yoshikawa H, Tuan RS, Nakamura N (2019) Enhanced repair of meniscal hoop structure injuries using an aligned electrospun nanofibrous scaffold combined with a mesenchymal stem cellderived tissue engineered construct. Biomaterials 192: 346-354.

Singh A, Goel SC, Gupta KK, Kumar M, Arun GR, Patil H, Kumaraswamy V, Jha S (2014) The role of stem cells in osteoarthritis: an experimental study in rabbits. Bone Joint Res 3: 32-37.

Sommaggio R, Pérez-Cruz M, Brokaw JL, Máñez R, Costa C (2013) Inhibition of complement component $\mathrm{C} 5$ protects porcine chondrocytes from xenogeneic rejection. Osteoarthritis Cartilage 21: 1958-1967.

Sommaggio R, Uribe-Herranz M, Marquina M, Costa C (2016) Xenotransplantation of pig chondrocytes: therapeutic potential and barriers for cartilage repair. Eur Cell Mater 32: 24-39.

Su J, Chen X, Huang Y, Li W, Li J, Cao K, Cao G, Zhang L, Li F, Roberts AI, Kang H, Yu P, Ren G, Ji W, Wang Y, Shi Y (2014) Phylogenetic distinction of iNOS and IDO function in mesenchymal stem cell-mediated immunosuppression in mammalian species. Cell Death Differ 21: 388-396.

Sullivan C, Barry F, Ritter T, O'Flatharta C, Howard L, Shaw G, Anegon I, Murphy M (2013) Allogeneic murine mesenchymal stem cells: migration to inflamed joints in vivo and amelioration of collagen induced arthritis when transduced to express CTLA4Ig. Stem Cells Dev 22: 3203-3213.

Tallheden T, Brittberg M, Peterson L, Lindahl A (2006) Human articular chondrocytes - plasticity and differentiation potential. Cells Tissues Organs 184: 55-67.

Tanaka Y (2005) Human mesenchymal stem cells as a tool for joint repair in rheumatoid arthritis. Clin Exp Rheumatol 33: 58-62.

Tatebe M, Nakamura R, Kagami H, Okada K, Ueda M (2005) Differentiation of transplanted mesenchymal stem cells in a large osteochondral defect in rabbit. Cytotherapy 7: 520-530.

Tawonsawatruk T, Spadaccino A, Murray IR, Peault B, Simpson HA (2012) Growth kinetics of rat mesenchymal stem cells from 3 potential sources: bone marrow, periosteum and adipose tissue. J Med Assoc Thai 95 Suppl 1: 189-197. 
Trivanović D, Kocić J, Mojsilović S, Krstić A, Ilić V, Djordjević IO, Santibanez JF, Jovcić G, Terzić M, Bugarski D (2013) Mesenchymal stem cells isolated from peripheral blood and umbilical cord Wharton's jelly. Srp Arh Celok Lek 141: 178-186.

Ullah I, Subbarao RB, Kim EJ, Bharti D, Jang SJ, Park JS, Shivakumar SB, Lee SL, Kang D, Byun JH, Park BW, Rho GJ (2016) In vitro comparative analysis of human dental stem cells from a single donor and its neuronal differentiation potential evaluated by electrophysiology. Life Sci 154: 39-51.

van Buul GM, Siebelt M, Leijs MJ, Bos PK, Waarsing JH, Kops N, Weinans H, Verhaar JA, Bernsen MR, van Osch GJ (2014) Mesenchymal stem cells reduce pain but not degenerative changes in a mono-iodoacetate rat model of osteoarthritis. J Orthop Res 32: 1167-1174.

Vista ES, Lau CS (2011) What about supplements for osteoarthritis? A critical and evidenced-based review. Int J Rheum Dis 14: 152-158.

Wang Q, Rozelle AL, Lepus CM, Scanzello CR, Song JJ, Larsen DM, Crish JF, Bebek G, Ritter SY, Lindstrom TM, Hwang I, Wong HH, Punzi L, Encarnacion A, Shamloo M, Goodman SB, WyssCoray T, Goldring SR, Banda NK, Thurman JM, Gobezie R, Crow MK, Holers VM, Lee DM, Robinson WH (2011) Identification of a central role for complement in osteoarthritis. Nat Med 17: 1674-1679.

Wang Y, Han ZB, Ma J, Zuo C, Geng J, Gong W, Sun Y, Li H, Wang B, Zhang L, He Y, Han ZC (2012) A toxicity study of multiple-administration human umbilical cord mesenchymal stem cells in cynomolgus monkeys. Stem Cells Dev 21: 1401-1408.

Wang K, Zhang T, Dong Q, Nice EC, Huang C, Wei Y (2013) Redox homeostasis: the linchpin in stem cell self-renewal and differentiation. Cell Death Dis 4: e537. DOI: 10.1038/cddis.2013.50.

Wang YH, Yang ZQ, Zhu SF, Gao Y (2018) Comparative study of methotrexate and human umbilical cord mesenchymal stem cell transplantation in the treatment of rheumatoid arthritis. J Biol Regul Homeost Agents 32: 599-605.

Wilke MM, Nydam DV, Nixon AJ (2007) Enhanced early chondrogenesis in articular defects following arthroscopic mesenchymal stem cell implantation in an equine model. J Orthop Res 25: 913-925.

Williams LB, Koenig JB, Black B, Gibson TW, Sharif S, Koch TG (2016) Equine allogeneic umbilical cord blood derived mesenchymal stromal cells reduce synovial fluid nucleated cell count and induce mild self-limiting inflammation when evaluated in a lipopolysaccharide induced synovitis model. Equine Vet J 48: 619-625.

Wu KC, Chang YH, Liu HW, Ding DC (2019) Transplanting human umbilical cord mesenchymal stem cells and hyaluronate hydrogel repairs cartilage of osteoarthritis in the minipig model. Ci Ji Yi Xue Za Zhi 31: 11-19.

Wu Y, Yang Z, Denslin V, Ren X, Lee CS, Yap FL, Lee EH (2020) Repair of osteochondral defects with predifferentiated mesenchymal stem cells of distinct phenotypic character derived from a nanotopographic platform. Am J Sports Med 38: 1857-1869.

Xia Q, Zhu S, Wu Y, Wang J, Cai Y, Chen P, Li J, Heng BC, Ouyang HW, Lu P (2015) Intra-articular transplantation of atsttrin-transduced mesenchymal stem cells ameliorate osteoarthritis development. Stem Cells Transl Med 4: 523-531.

Xia H, Liang C, Luo P, Huang J, He J, Wang Z, Cao X, Peng C, Wu S (2018) Pericellular collagen I coating for enhanced homing and chondrogenic differentiation of mesenchymal stem cells in direct intra-articular injection. Stem Cell Res Ther 9: 174. DOI: 10.1186/s13287-018-0916-z.

Xia T, Yu F, Zhang K, Wu Z, Shi D, Teng H, Shen J, Yang X, Jiang Q (2018b) The effectiveness of allogeneic mesenchymal stem cells therapy for knee osteoarthritis in pigs. Ann Transl Med 6: 404. DOI: 10.21037/atm.2018.09.55.

Xing D, Kwong J, Yang Z, Hou Y, Zhang W, Ma B, Lin J (2018) Intra-articular injection of mesenchymal stem cells in treating knee osteoarthritis: a systematic review of animal studies. Osteoarthritis Cartilage 26: 445-461.

Yamaguchi S, Aoyama T, Ito A, Nagai M, Iijima H, Tajino J, Zhang X, Kiyan W, Kuroki H (2016) The effect of exercise on the early stages of mesenchymal stromal cell-induced cartilage repair in a rat osteochondral defect model. PLoS One 11: e0151580. DOI: 10.1371/journal.pone.0151580.

Yang X, Zhu TY, Wen LC, Cao YP, Liu C, Cui YP, Meng ZC, Liu H (2015) Intraarticular injection of allogenic mesenchymal stem cells has a protective role for the osteoarthritis. Chin Med J (Engl) 128: 2516-2523.

Yeh HY, Lin TY, Lin CH, Yen BL, Tsai CL, Hsu SH (2013) Neocartilage formation from mesenchymal stem cells grown in type II collagen-hyaluronan composite scaffolds. Differentiation 86: 171-183.

Yin $\mathrm{H}$, Wang $Y$, Sun Z, Sun X, Xu Y, Li P, Meng H, Yu X, Xiao B, Fan T, Wang Y, Xu W, Wang A, Guo Q, Peng J, Lu S (2016) Induction of mesenchymal stem cell chondrogenic differentiation and functional cartilage microtissue formation for in vivo cartilage regeneration by cartilage extracellular matrix-derived particles. Acta Biomater 33: 96-109.

Yuan T, Li K, Guo L, Fan H, Zhang X (2011) Modulation of immunological properties of allogeneic mesenchymal stem cells by collagen scaffolds in cartilage tissue engineering. J Biomed Mater Res A 98: 332-341.

Zhang S, Chu WC, Lai RC, Lim SK, Hui JH, Toh WS (2016) Exosomes derived from human embryonic mesenchymal stem cells promote osteochondral regeneration. Osteoarthritis Cartilage 24: 2135-2140.

Zhang Y, Liu S, Guo W, Wang M, Hao C, Gao S, Zhang $X$, Li X, Chen M, Jing X, Wang Z, Peng J, Lu S, Guo Q (2018) Human umbilical cord Wharton's jelly mesenchymal stem cells combined with an acellular 
cartilage extracellular matrix scaffold improve cartilage repair compared with microfracture in a caprine model. Osteoarthritis Cartilage 26: 954-965.

Zhang Q, Li Q, Zhu J, Guo H, Zhai Q, Li B, Jin Y, He X, Jin F (2019) Comparison of therapeutic effects of different mesenchymal stem cells on rheumatoid arthritis in mice. PeerJ 7: e7023. DOI: 10.7717/ peerj.7023.

\section{Discussion with Reviewer}

Stephan Zeiter: Is there a need to have more standardized preclinical models?

Authors: Yes. An extensive review of the field leads to the understanding that a higher level of standardization of some preclinical models would help the progress of MSC-based therapies towards clinical application. This is especially relevant for large animal models that may currently lack the level of characterization and tools available in small animal models. Nevertheless, large animal models are still of high interest because of their greater similarity to the human patient. The progress of both preclinical and clinical studies should help identifying and improving the most informative preclinical models.

Editor's note: The Scientific Editor responsible for this paper was Martin Stoddart. 